\title{
Red-sequence galaxies with young stars and dust: the cluster Abell 901/902 seen with COMBO-17^
}

\author{
C. Wolf ${ }^{1}$, M. E. Gray ${ }^{2}$, and K. Meisenheimer ${ }^{3}$ \\ 1 Department of Physics, Denys Wilkinson Bldg., University of Oxford, Keble Road, Oxford, OX1 3RH, UK \\ e-mail: cwolf@astro.ox.ac.uk \\ 2 School of Physics and Astronomy, University of Nottingham, Nottingham, NG7 2RD, UK \\ 3 Max-Planck-Institut für Astronomie, Königstuhl 17, 69117 Heidelberg, Germany
}

Received 7 June 2005 / Accepted 3 August 2005

\section{ABSTRACT}

We report the discovery of a rich component of dusty star-forming galaxies contaminating the red-sequence in the supercluster system comprising Abell 901a, 901b and A902 at redshift $\sim 0.17$. These galaxies do not fit into the colour-density relation, because their preferred habitat is different from that of regular red-sequence galaxies, which are typically dust-poor, old and passively evolving. The dusty red galaxies prefer the medium-density outskirts of clusters while being rare in both the low-density field and the high-density cluster cores. This new result is based on the information content in the medium-band photometry of the COMBO-17 survey. The photo- $z$ accuracy of the $\sim 800$ brightest cluster galaxies is $<0.01$ and of the order of the velocity dispersion of the cluster. This enables us to select a rich and clean cluster sample, in which we can trace age-sensitive and dust-sensitive spectral features independently with the detailed medium-band SED data. We find the red colour of the dusty galaxies to be a result of dust extinction combined with relatively old stellar ages. We speculate that the dusty red galaxies could either be a product of minor mergers between established old red cluster galaxies with infalling blue field galaxies, or mark a period in the internal transformation of blue field galaxies into red cluster galaxies, which is triggered by the environmental influences experienced during cluster infall.

Key words. surveys - techniques: photometric - methods: data analysis - galaxies: clusters: general - galaxies: evolution

\section{Introduction}

It has been known for decades that massive galaxies in clusters tend to have spheroid-dominated morphologies (Dressler 1980; Dressler et al. 1997) and a paucity of recent star formation (Butcher \& Oemler 1984; Lewis et al. 2002). Dressler's morphology-density relation can be seen as a result of an environmental influence on galaxy properties, whereby a higher density of galaxies supresses star formation and leads to pressure-supported morphologies via some still controversial physical mechanism. Alternatively, the galaxies in denser regions could just be exhibiting a more mature state because they were the earliest to collapse and evolved most quickly. Both concepts could explain the observation that the percentage of red old galaxies increases with the local galaxy density, while that of blue young galaxies decreases correspondingly.

So far, it appears that clusters conform with rather universal trends of evolution in colour-magnitude and colour-density relations (McIntosh et al. 2005), if only a simple bimodal distinction of red vs. blue galaxies is considered, which is most simply translated into non-star-forming vs. star-forming galaxies. However, from ISOCAM observations Coia et al. (2005)

^ Appendices A and B are only available in electronic form at http://www. edpsciences.org have concluded that some of the red-sequence galaxies in the cluster $\mathrm{Cl} 0024+1654$ are undergoing bursts of star formation while their red colour is due to their very dusty nature.

In this paper, we present striking evidence that the redsequence of the clusters Abell 901/902 is highly contaminated by galaxies which are not just old and passively evolving. Instead we find a large population of dusty star-forming galaxies in the red sequence, which do not fit into the simple colourdensity relation. These dusty red cluster galaxies can neither be considered a subset of typical red galaxies nor a subset of typical blue galaxies, because their spatial distribution is inconsistent with either one of them.

It is possible that the dusty red star-forming galaxies may be in the process of transformation from typical blue field galaxies to typical red cluster galaxies. But it is equally conceivable that the newly identified population is undergoing just a particular phase in a more complicated evolutionary picture. Miller \& Owen (2002) found star-forming galaxies with large amounts of extinction to be concentrated more centrally in clusters than star-forming galaxies in general, suggesting an environmental effect. Coia et al. (2005) have studied dusty starbursts in several clusters and found great variety in abundance even when clusters of similar mass and distance were investigated. They found dusty starbursts most often in clusters with 
complex dynamics that were suggestive of sub-cluster mergers. In fact, our target Abell 901/902 is a dynamically very complex supercluster environment.

A variety of physical processes are expected to play an important role in the environmentally triggered transformation from star-forming galaxies to red old spheroidal systems: e.g., major mergers between galaxies of comparable size (Barnes 1992); impulsive encounters where galaxies move at too high a relative speed to merge yet are dynamically heated and sometimes eventually disrupted (minor interactions and galaxy harassment; Moore et al. 1999); ram-pressure stripping, where a galaxy's gas content is stripped off as it passes through the hot, extended gas characteristic of large groups and galaxy clusters (Gunn \& Gott 1972); and suffocation, where a galaxy loses its warm gas envelope as a reservoir for future cooling and gas accretion (Larson et al. 1980). Bekki (1999) suggested that tidal forces exerted by a cluster onto an infalling group could trigger nuclear starbursts with no merging being involved.

Recent results from a variety of authors indicate that the transformation of massive star-forming galaxies into spheroidal non-star forming galaxies takes place in galaxy groups, rather than in cluster environments (Kodama et al. 2001; Balogh et al. 2002). Therefore, it is necessary not just to study the cores of massive clusters but to explore the properties of galaxies out to large radii, towards densities characteristic of the field. However, detailed studies of galaxy populations in clusters have been very challenging, because without exhaustive spectroscopy it is difficult to identify which galaxies are truly embedded in the cluster environment.

An alternative route presented here for the first time involves the use of high-precision photometric redshifts such as those provided by the medium-band survey COMBO-17 (Wolf et al. 2001). These permit a sufficiently clean selection of cluster galaxies with very little field contamination. The detailed medium-band SEDs contain a wealth of spectral information which allows simultaneous decoupling of redshift, age and dust reddening using purely optical photometric data.

One of the COMBO-17 fields contains a complex supercluster environment which is very suitable to study the evolutionary phenomena near galaxy clusters and test the role of the physical mechanisms listed above. This is the A901 field of COMBO-17 which contains the clusters Abell 901a, 901b and 902, as well as some associated groups, all at redshift $\sim 0.165$ and within a projected area of $3.5 \times 3.5 \mathrm{Mpc} / h$. This field has already been subject of weak lensing studies in two and three dimensions using the COMBO-17 data (Gray et al. 2002; Taylor et al. 2004). A first study of the cluster population itself investigated the relation of galaxy colours to the darkmatter density as inferred from weak lensing maps (Gray et al. 2004). The field has since been observed with XMM-Newton, Spitzer, HST/ACS and GALEX.

In this paper, we examine the galaxy population in this cluster complex using the optical data from COMBO-17 and taking advantage of accurate photometric redshifts which have errors below 0.01 for most objects studied. We exploit the 17-filter SED data to differentiate between dust-free and obscured galaxies, and thus identify dusty galaxies in the redsequence from our multi-band photometry alone. As a result, we are able to identify the true old population unambiguously, and investigate the properties of the dusty red-sequence galaxies. The full COMBO-17 data set provides us with a field comparison.

The paper is structured as follows: in Sect. 2, we present the COMBO-17 data and their characteristics. Section 3 reports on the galaxy sample selected from the COMBO-17 survey catalogue. In Sect. 4 we compile our analysis of the cluster population characterizing the several types of galaxies and deriving a type-density relation. Section 5 presents a discussion of our results, and finally we summarize in Sect. 6.

Throughout the paper we use $H_{0}=h \times 100 \mathrm{~km} /(\mathrm{s} \mathrm{Mpc})$ in combination with $\left(\Omega_{\mathrm{m}}, \Omega_{\Lambda}\right)=(0.3,0.7)$ and use $h=1$ when citing luminosities in the text. Both apparent and absolute magnitudes are reported in the Vega system and are total object magnitudes. All rest-frame quantities are corrected for Galactic foreground reddening, while apparent magnitudes and observed-frame colours are used as observed. Rest-frame colour indices are always labelled as such, while observedframe indices have no special labels.

\section{Data}

\subsection{The multi-band SEDs from $C O M B O-17$}

The COMBO-17 survey has measured accurate photometric redshifts for more than 30000 galaxies in three different fields, including the Chandra Deep Field South (Wolf et al. 2004) and the field of Abell 901/902 which is analysed in this paper. Photometry in 5 broad ( $U B V R I)$ and 12 medium-bands is used to classify objects into normal stars, white dwarfs, galaxies and QSOs (more generally type-1 AGN) and to estimate redshifts for extragalactic objects. Quasars are clearly identified when the contribution of AGN light to the SED is sufficient to leave recognizable traces in the 17-band SED data. Comparisons with spectroscopic redshifts and simulations have suggested the mean error of COMBO-17 redshifts to be $\sigma_{z} /(1+$ $z)<0.01$ for galaxies with $R<20$, while fainter galaxies have higher redshift errors. We found the $90 \%$ completeness limit for galaxies at $z<1$ to be $R \approx 23$, fainter than the samples analyzed later in this paper.

The COMBO-17 filters have central wavelengths ranging from observed-frame 365 to $915 \mathrm{~nm}$ and allow us to derive luminosities for cluster galaxies in A901 between $310 \mathrm{~nm}$ and $740 \mathrm{~nm}$ rest-frame wavelength directly from observed photometry. We obtain luminosities by placing the best-fitting template SED into the observed 17-filter spectrum and integrating the template over the efficiency curve of the desired redshifted rest-frame band. However, the analysis here relies mostly on observed-band photometry to eliminate impacts of errors in the derivation of rest-frame quantities. All luminosities refer to total object photometry, while the colours and SED shapes are determined from seeing-adaptive aperture photometry probing the same physical section of any object in all 17 bands. The faintest objects in the sample contain $\sim 60 \%$ of their total light in the apertures, but the few most luminous and largest galaxies emit only $\sim 20 \%$ of the total in the aperture. As a result, the SEDs of larger galaxies are more dominated by nuclear light. 
All survey details regarding observations, data reduction, classification, redshift estimation, rest-frame properties, completeness, accuracy and reliability of the object catalogue data are discussed in great detail in Wolf et al. (2004). Here, we only repeat a discussion of the galaxy templates as this is relevant for the analysis of the galaxy population in Abell 901/902.

\subsection{Galaxy templates and SED parameters}

The galaxy template library used in COMBO-17 contains a grid of synthetic spectra based on the PEGASE code (Fioc \& Rocca-Volmerange 1997) for population synthesis models. The templates span a two-dimensional grid with a range of ages calculated by the PEGASE code and a range of extinction levels which we applied as external screens to the SEDs delivered by PEGASE. The setup for PEGASE uses standard parameters suggested by the codes SSPs and scenarios with a Kroupa (1993) IMF and no extinction. The star formation history follows an exponential decay law with a time constant of $\tau=1 \mathrm{Gyr}$. The SEDs are calculated by PEGASE for various time steps since the beginning of the first star formation. As templates we use 60 spectra which span ages (more precisely, time since onset of star formation) from $50 \mathrm{Myr}$ to $15 \mathrm{Gyr}$. All templates are then extinguished with six different equidistant degrees of reddening in the interval of $E_{B-V}=[0.0,0.1, \ldots 0.5]$. The reddening law of our choice is the SMC law from the 3-component model by Pei (1992). We decided to use this law because it provided a reasonably good match between the restframe UV templates and the observed SEDs of galaxies with known redshift. Note that the detailed choice of extinction law has no effect on the observed SEDs of galaxies with $z<0.6$, and hence certainly not on the cluster A901.

We tune initial model metallicities to give almost solar metallicity for old populations typical of local $\mathrm{L}^{*}$ spheroidal galaxies. It is worth noting, however, that the well-known age/metallicity degeneracy is actually helpful in this case: Mismatches between real galaxy and template metallicities can be compensated for by modest changes in template age, while yielding still accurate estimates of redshift and SED shape.

\subsection{The Abell $901 / 902$ field}

The COMBO-17 observations cover an area of 31.5 $\times 29$ '.7 at full depth, centered on $(\alpha, \delta)_{2000}=\left(9^{\mathrm{h}} 56^{\mathrm{m}} 17^{\lessgtr} 3,-10^{\circ} 01^{\prime} 11^{\prime \prime}\right)$, which is $(l, b)_{\text {gal }}=(248,+34)$. The interstellar foreground reddening is estimated at $E_{B-V}=0.06$ (Schlegel et al. 1998), which is taken into account for fitting templates to the observed photometry and deriving the correct photometric redshift.

At the cluster redshift $z=0.165$, the angular scale is $2.0 / h \mathrm{kpc}$ per arcsec. Assuming $h=0.7$ the full field covers an area of $5.35 \times 5.05 \mathrm{Mpc}$. This area contains three pronounced cluster cores called Abell 901a, 901b and 902, as well as various associated groups. A deep WFI image of the field has been published in Gray et al. (2002).

The field contains 11009 objects brighter than $R=23$, of which $98 \%$ are classified by COMBO-17. Due to its relatively low galactic latitude of $\sim 34^{\circ}$, the field contains a large proportion of stars. The COMBO-17 classifier found 2091 stars and 13 white dwarfs at $R<23$. A number of bright stars reduce the effective field slightly and constrain some multi-wavelength follow-up observations. The brightest star in the field is an F8 star with $V=9.6$ named PPM $192892=$ SAO $137231=$ BD-09 2962. At $\lambda>1 \mu$ the brightest object is an M8 Mira variable (Kirkpatrick et al. 1997, IRAS 09540-0946 = GSC 0547901188 ) with $V \approx 15$ and $K=5.75$ (measured by 2MASS).

After eliminating stars, QSOs, objects too strange to be classified and objects with bad flags, we have 7992 galaxies left with estimated redshifts and $R<23$ to search for members of the cluster A901/902.

\subsection{Spectroscopy}

Spectra of supercluster galaxies were obtained using the 2dF instrument on the AAT in March 2002 and March 2003. Details of these observations are to be published in Gray et al. (in preparation). A total of 89 galaxies were targetted using the $1200 \mathrm{~B}$ grating in a single fibre configuration during the 2002 run, of which 64 were within $0.15<z<0.18$. Three fibre configurations using the lower resolution $600 \mathrm{~V}$ grating were obtained during the 2003 run targetting 368 objects, with 47 repeated from the previous run. The primary selection function targetted galaxies selected by photometric redshift to be within the supercluster redshift and having $R<20$, with additional fibres being allocated to secondary targets (QSOs or non-cluster galaxies) when available. However, the limitations on close packing of fibres meant that the highest-density regions of the clusters were less well sampled, thereby missing a number of the brightest galaxies. The fibers measure $2^{\prime \prime}$ in diameter and measure light from very similar fractions of any object to the photometric apertures.

Redshifts were obtained by two independent means, by line profile fitting of the $\mathrm{Ca} \mathrm{H}$ and $\mathrm{K}$ feature in absorption and by cross-correlation with template spectra using the XCSAO task within IRAF. The two measures agreed well, with $\left\langle\Delta_{z}\right\rangle=0.00149 \pm 0.00006$. In total, spectra were obtained for 407 unique objects including various stars and QSOs that were targets of different interest. After eliminating several galaxies due to data quality issues, we have 249 galaxy spectra within the redshift range of the supercluster for statistical analysis.

\section{The galaxy sample}

\subsection{Selecting the cluster population at $M_{V}<-17$}

Figure 1 shows a redshift-magnitude diagram of the galaxy sample focussing on a redshift interval encompassing the target clusters. The bulk of the bright cluster population can be clearly seen at $z \approx 0.17$, reaching up to apparent magnitudes of $R \approx 16$. Fainter than $R=20$ the photometric redshift errors slowly start to widen the cluster distribution, which makes it harder to disentangle cluster members from field contaminants. In fact, at the bright end $(R<20)$ we expect a photometric redshift error of $\sigma_{z}<0.01$, while at $R=23$ we expect $\sigma_{z} \approx 0.03$. 

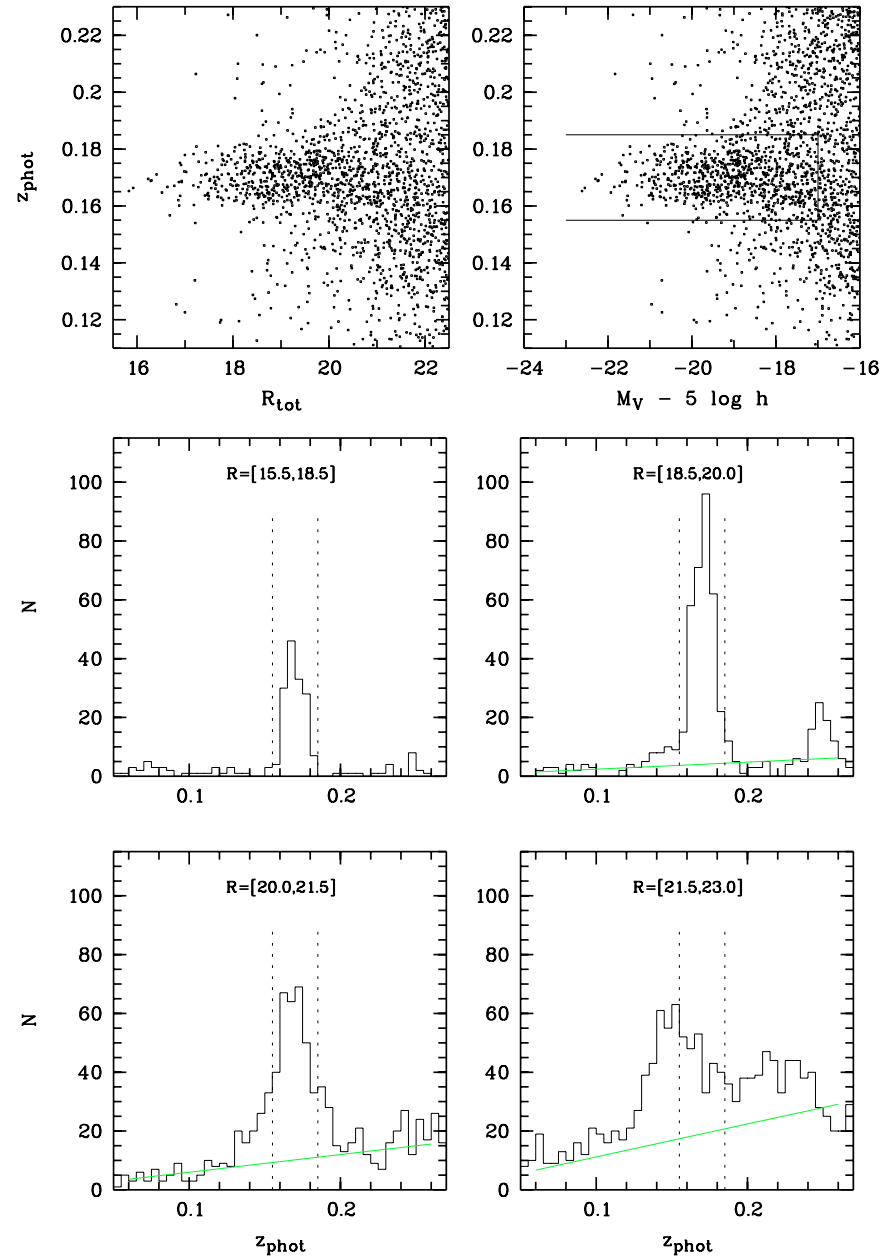

Fig. 1. Bright cluster galaxies: top row, left: photometric redshift vs. apparent magnitude for the galaxy sample in the A901 field with the cluster at $z \approx 0.17$. Top row, right: redshift vs. absolute magnitude. The lines show the selection for the cluster sample used in the following analysis. Bottom four panels: redshift histograms in apparent magnitude bins. The dashed lines show the redshift interval for the adopted cluster sample. At $R>21$ the errors in photometric redshift start to broaden the cluster distribution and bias it to lower redshift. A rough estimate for the field contribution is shown in grey.

We first decide on a redshift cut for the cluster sample by looking at the redshift distribution in a bright sample with small photo- $z$ errors, before defining a deeper sample on the basis of this redshift cut. For this, we use an iterative approach to determine the mean and rms redshift of the cluster population. We settled on a redshift cut of $z_{\text {phot }}=[0.155,0.185]$ at $R<20$. This sample contains 482 galaxies with a redshift distribution of

$\left\langle z_{\text {phot }}\right\rangle=0.1703 \pm 0.0063$,

suggesting 99\% completeness within the redshift cut if the distribution is Gaussian. The scatter translates into a velocity dispersion from photometric redshifts of $\sigma_{c z} \approx 1900 \mathrm{~km} \mathrm{~s}^{-1}$, which is a combination of velocity scatter among cluster galaxies and photo- $z$ errors. The velocity scatter in the spectroscopic sample is $\sim 1250 \mathrm{~km} \mathrm{~s}^{-1}$, and requires convolution with a photo- $z$ error of $\sigma_{z} /(1+z)=0.004$ to produce the full scatter. While this is only the relative redshift scatter within the
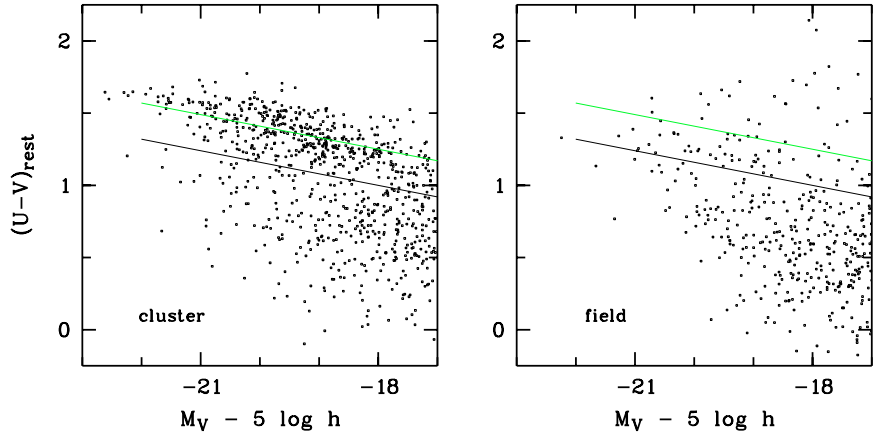

Fig. 2. Rest-frame colour-magnitude diagrams: cluster (left) versus field (right) with cluster red-sequence fit (grey line) and ButcherOemler style red-sequence cut (black line).

cluster, the full photo- $z$ accuracy is slightly lower: we find the mean redshift in the spectroscopic sample to be 0.165 , which is offset by 0.005 from the mean photo- $z$ (see also Fig. A.1).

We now define a deeper cluster sample for the following analysis and keep the redshift range at $z_{\text {phot }}=[0.155,0.185]$. The fainter we go, the lower the completeness of the cluster sample will be as more and more galaxies are scattered outside the redshift interval due to increasing photo- $z$ errors. However, widening the interval would clearly include a large amount of field contaminants, which we would like to avoid. For this paper, we settle to applying a luminosity cut at $M_{V}<-17$, which corresponds to $R \approx 21.5$. The low redshift errors in this bright regime help to keep field contamination low and cluster completeness high. At $R=21.5$ we expect redshift errors of $\sigma_{z} \approx 0.015$, which would make the sample still $68 \%$ complete at its luminosity limit.

Hereby, we select 795 galaxies in a comoving volume of $\sim 1575(\mathrm{Mpc} / h)^{3}$. Although the clusters occupy only a tiny fraction of this volume, the sample consists mostly of cluster galaxies due to their large overdensity. We split the sample into red-sequence and blue cloud following the procedure explored by Bell et al. (2004) on COMBO-17 galaxies. We apply a cut parallel to the colour-magnitude relation of the red-sequence, which evolves with redshift. From the COMBO-17 sample Bell et al. (2004) have suggested two alternative cuts, one determined internally from COMBO-17 and another one which includes measurements from the local Universe. Here, we use the COMBO-17 internal cut defined by

$(U-V)_{\mathrm{rest}}>(U-V)_{\mathrm{CMR}}-0.25$,

where

$(U-V)_{\mathrm{CMR}}=1.48-0.4 z-0.08\left(M_{V}-5 \log h+20\right)$.

This CMR fit matches the red-sequence of A901/902 at $z=$ 0.165 , and splits the cluster sample into 462 red-sequence galaxies and 333 blue cloud galaxies (see Fig. 2).

\subsection{The field sample and field contamination}

We select a low-redshift field comparison sample for two purposes: we would like to estimate the field contamination in the redshift slice of the cluster sample from the space density of 

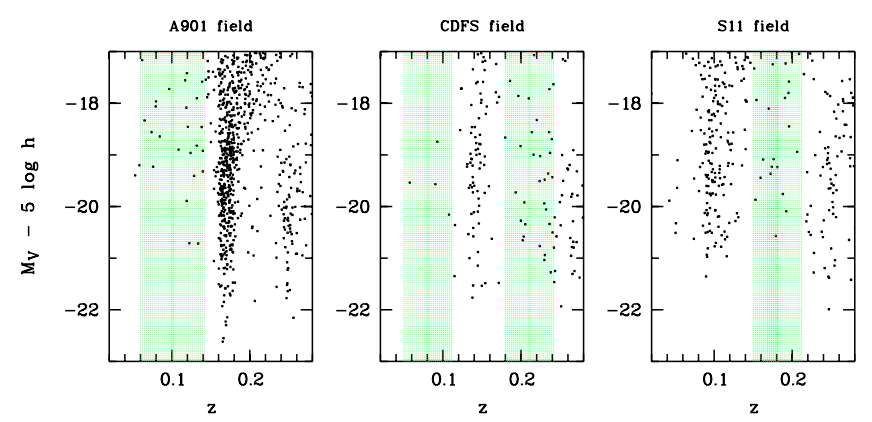

Fig. 3. Selection of field galaxy sample: luminosity-redshift diagrams of red-sequence galaxies show rich groups and clusters in all three COMBO-17 fields. We exclude them to select a field sample (shaded area) from the low-redshift domain of all fields.

galaxies outside the cluster using the same luminosity cut; and we would like to compare the properties of the cluster population to that of the field.

We would like the field sample to have a similar mean redshift as the cluster to suppress possible influence from any evolutionary trends and consider all three COMBO-17 fields. We investigate the redshift range of $z=[0.05,0.25]$, where the photo- $z$ catalogue is more than $90 \%$ complete at $M_{V}=-17$ for all galaxy SED types. We use the same luminosity cut and determine the number of galaxies and the volume they are drawn from, hence obtaining a space density.

We want to exclude strong local overdensities, such as the Abell clusters at $z \sim 0.11$ in the $S 11$ field and at $z \sim 0.17$ in the A901 field as well as some rich groups at $z \sim 0.25$. Figure 3 shows overdensities in redshift space for all three COMBO-17 fields as indicated only by the strongly clustered red-sequence galaxies. Avoiding obvious overdensities we adopt the redshift ranges shaded in grey. The field sample has a mean redshift of $\left\langle z_{\text {field }}\right\rangle=0.172$, which is very close to the redshift of A901. It contains 385 galaxies from a volume of $\sim 9750(\mathrm{Mpc} / h)^{3}$.

The volume of the cluster sample at $z=[0.155,0.185]$ is more than six times smaller, and contains an estimated field contamination of 62 galaxies $^{1}$. This implies that statistically one in 13 out of the 795 galaxies in the cluster sample would really be in the physically unrelated field, somewhere along the line-of sight within $\pm 50 / h \mathrm{Mpc}$ from the cluster center. Since red-sequence galaxies are intrinsically more clustered than blue galaxies, we split the contamination assessment by galaxy colour by applying the evolving red-sequence cut to the field sample. Our best estimate is that 13 out of 468 redsequence galaxies (3\%), and 49 out of 327 blue-cloud galaxies $(15 \%)$ are field contaminants. No attempt is made in this paper to subtract the field contamination from any numbers reported for the cluster.

1 We note, that e.g. the photo- $z$ selection for cluster galaxies at $z=$ 0.41 based on pure broad-band photometry in Kodama et al. (2001) used a 5.33 times wider redshift bin, which led to a 33 times higher estimated field contamination: the physical size of their studied area was $\sim 3 \times$ larger as well, and the luminosity cut was marginally fainter.
Table 1. Mean properties of the three galaxy SED class samples.

\begin{tabular}{lrrr}
\hline \hline property & Dust-free old Dusty red-seq & Blue cloud \\
\hline$N_{\text {galaxy }}$ & 294 & 168 & 333 \\
$N_{\text {field contamination }}$ & 6 & 7 & 49 \\
$N_{\text {spectra }}$ & 144 & 69 & 36 \\
\hline$z_{\text {spec }}$ & 0.1646 & 0.1646 & 0.1658 \\
$\sigma_{c z} /(1+z) /\left(\mathrm{km} \mathrm{s}^{-1}\right)$ & 939 & 1181 & 926 \\
$z_{\text {spec,N }}$ & 0.1625 & 0.1615 & $\mathrm{~N} / \mathrm{A}$ \\
$z_{\text {spec,S }}$ & 0.1679 & 0.1686 & $\mathrm{~N} / \mathrm{A}$ \\
$\sigma_{c z, \mathrm{~N}} /(1+z) /\left(\mathrm{km} \mathrm{s}^{-1}\right)$ & 589 & 597 & $\mathrm{~N} / \mathrm{A}$ \\
$\sigma_{c z, \mathrm{~S}} /(1+z) /\left(\mathrm{km} \mathrm{s}^{-1}\right)$ & 522 & 546 & $\mathrm{~N} / \mathrm{A}$ \\
$\log \left(\Sigma_{10}(\mathrm{Mpc} / h)^{2}\right)$ & 2.188 & 1.991 & 1.999 \\
$E W_{\mathrm{e}}(\mathrm{OII}) / \AA$ & $\mathrm{N} / \mathrm{A}$ & $4.2 \pm 0.4$ & $17.5 \pm 1.5$ \\
$E W_{\mathrm{a}}(\mathrm{H} \delta) / \AA$ & $2.3 \pm 0.5$ & $2.6 \pm 0.5$ & $4.5 \pm 1.0$ \\
\hline age/Gyr & 6.2 & 3.5 & 1.2 \\
$E_{B-V}$ & 0.044 & 0.212 & 0.193 \\
$(U-V)_{\text {rest }}$ & 1.372 & 1.293 & 0.670 \\
$M_{V, \text { rest }}$ & -19.31 & -19.18 & -18.47 \\
\hline$B-R$ & 1.918 & 1.847 & 1.303 \\
$V-I$ & 1.701 & 1.780 & 1.290 \\
$R-I$ & 0.870 & 0.920 & 0.680 \\
$U-420$ & 0.033 & -0.079 & -0.377 \\
$420-464$ & 0.537 & 0.602 & 0.560 \\
$464-518$ & 0.954 & 0.827 & 0.490 \\
$604-646$ & 0.356 & 0.339 & 0.238 \\
$753-815$ & 0.261 & 0.274 & 0.224 \\
\hline & & & \\
& & & \\
& & &
\end{tabular}

\subsection{Galaxies as a 2-parameter family}

To first order, galaxy SEDs are often considered to form a 1-parameter family, running from old populations in red spheroidal galaxies to young starbursts in blue galaxies (Connolly et al. 1995). Also, plenty of photometric redshift codes use a 1-parameter family of templates. In COMBO-17, such an approach was taken until 2002, by when it became clear that this was inappropriate at least for the galaxies in A901. Using the Kinney et al. (1996) template spectra, $\sim 1 / 4$ of the bright galaxies in the cluster had redshift errors up to $\lesssim 0.1$ and would have been left out from the study presented here.

However, using the 2-D template set outlined above the redshifts of virtually all cluster galaxies were correctly estimated. The 2-parameter aspect of the galaxy family is of central importance, to accommodate spectra with different curvature while keeping the same overall colour. This issue becomes apparent when looking at colour-colour diagrams of the cluster in Fig. 4. Here, the panels on the left-hand side show a narrow distribution of galaxy colours in observed-frame $B-R$ over $U-I$. The approximate position of the gap between red-sequence and blue cloud galaxies is given by a line cutting across the distribution, although the gap is washed out by the CMR slope in 

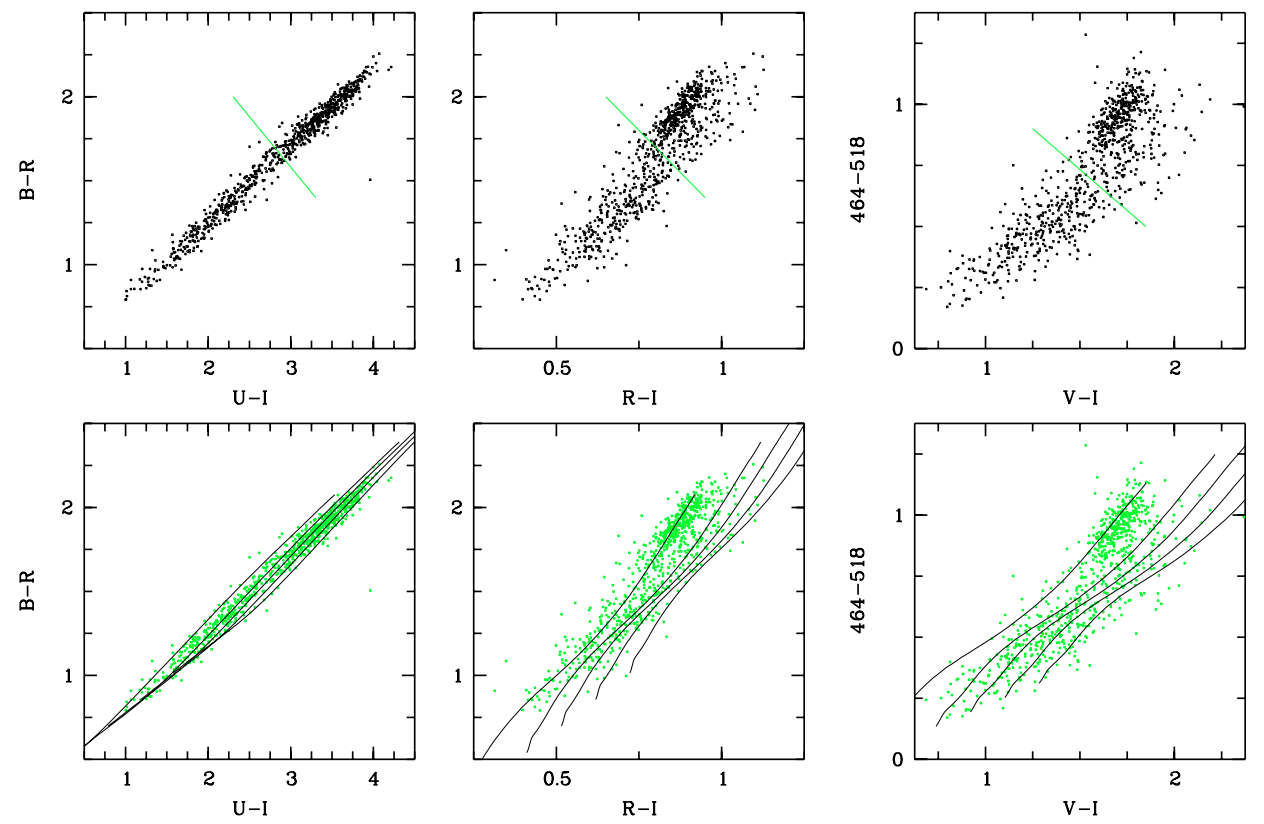

Fig. 4. Observed-frame colour-colour digrams: top row, left: two observed-frame colour-indices, each straddling the $4000 \AA$-break, show galaxies as a 1-parameter family. The grey line roughly indicates the location of the gap in the colour bimodality. Top row, center: when one colour index does not encompass the $4000 \AA$-break, the galaxies fan out into a wider range. Top row, right: a medium-band colour index probing the $4000 \AA$ A-break. Bottom row: template colours (black lines) are plotted over the galaxy sample (grey points). The left-most line is an age sequence from $50 \mathrm{Myr}$ (bottom left) to $15 \mathrm{Gyr}$ (top right) without dust reddening. The four other lines show colours for the same age sequence with reddening of $E_{B-V}=[0.2,0.3,0.4,0.5]$ omitting the line at 0.1 for clarity.

combination with a wide luminosity interval collapsed in this plot. The center panels show galaxies more spread out in the colour plane $B-R$ over $R-I$, and suggest the existence of a distinctive elongated clump at the red end, almost detached from the main, larger and less concentrated, distribution.

The observed-frame $B-R$ encloses the $4000 \AA$-break at $z \sim$ 0.16 as does the colour index $U-I$, in contrast to the colour index $R-I$ which probes the red end of the observed stellar spectrum. The spread in $R-I$ colour at fixed $B-R$ colour means that the $4000 \AA$-break is not a safe predictor for the colour at longer (or even shorter) wavelengths. Since the 4000 Å-break is mostly driven by the mean stellar age of a galaxy, the spread in colours requires another important factor.

A comparison with the age $\times$ dust template grid shows dust reddening to be a possible second independent factor in galaxy colours. The pronounced red clump coincides with templates of old age and no dust reddening, and is hereafter referred to as dust-free old clump. It may represent typical spheroidal galaxies in the cluster, while galaxies with redder $R-I$ colours at fixed $B-R$ value correspond to dust-reddened objects with younger mean stellar age. This interpretation of galaxies as a 2-D age $\times$ dust family can further be tested with the COMBO-17 medium-band colours. In the right-hand panels of Fig. 4 we see again the clear 2-D parameter family. Colour indices from neighboring medium-band filters probe small-scale variations in the SED. Near the $4000 \AA$-break these differ between galaxies that are red because of age and those that are red because of dust. We note, that medium-band indices where both filters are redwards of the $4000 \AA$-break do not differentiate age and dust, and galaxies appear again as 1-D families.

\subsection{What is in the red sequence? Provisional galaxy SED classification}

The colour-colour diagrams already suggested that we have two contributions to the red-sequence of Abell 901/902: a pronounced dust-free old clump; and a more widely scattered collection of galaxies with higher dust reddening and lower mean stellar age. We thus try go beyond a simple classification of galaxies into a red-sequence and a blue cloud from their colour bimodality. We want to split the red-sequence itself into two different types in order to investigate their possibly different properties in terms of distribution in space, luminosity, their environment and their spectral properties. We try to separate the pronounced dust-free old clump from dusty red-sequence galaxies by cutting in template parameter space rather than colour space. We find ad-hoc that a simple reddening cut at $E_{B-V}=0.1$ delivers a reasonably clean separation between the two features (see Fig. 5).

For the purpose of this paper, we will now provisionally classify the galaxy population into three categories:

1. As usual, on the blue side of the red-sequence cut, we have the blue cloud of star-forming galaxies.

2. The dust-free old clump are red-sequence galaxies with $E_{B-V}<0.1$. We consider them an improved selection of old stellar populations with much less contamination from dust-reddened galaxies than a regular red-sequence cut (at least in Abell 901/902).

3. The dusty red-sequence are red-sequence galaxies with $E_{B-V}>0.1$. We consider them dusty contaminants to the red-sequence or an older-age tail of the blue cloud reaching across the red-sequence, and we are surprised by their large 

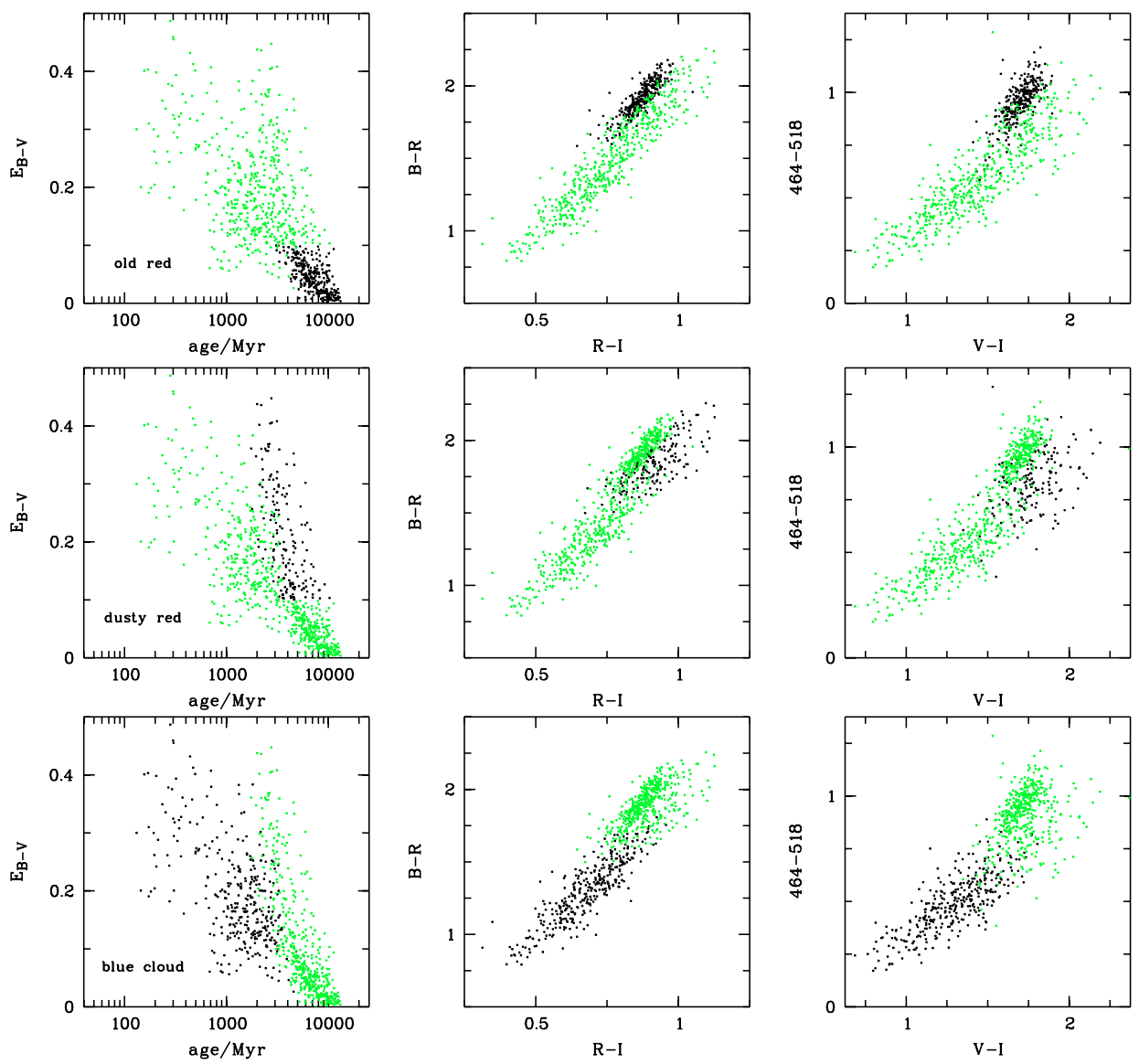

Fig. 5. Three galaxy types: left: stellar age vs. $E_{B-V}$ from estimated template parameters. Center/Right: broad-band/medium-band colour. Top row: dust-free old clump in the red-sequence. Middle row: dusty population in the red-sequence. Bottom row: the blue cloud.

number. We investigate their nature and relation to the other galaxy types further in this paper.

This classification is an improvement over the simple bimodality split of the galaxy population and motivated by both an interest in analyzing dust-reddened contaminants of the redsequence, and a desire to have a cleaner sample of old, passively evolving stellar populations. However, it is no ideal classification from a physical point of view. Eventually, we would rather want to understand how the mean stellar age or the total star formation rate depend on galaxy environment. But the total star formation rate can not be measured from optical data alone as it does not provide any clues to how much star formation is hidden by dust. We are looking forward to ongoing observations with Spitzer which will provide a full account of star formation in this cluster. In this paper, we will investigate the properties of the three galaxy classes as outlined above.

\section{Analysis of galaxy types}

\subsection{Mean SEDs for three classes}

We would now like to investigate the average spectral properties among the three galaxy SED classes just defined. We will ignore the age-metallicity degeneracy here, since a detailed discussion of ages and star formation histories is beyond the scope of this paper, and the degeneracy will not matter much for the overall shape of continuum spectra and colour indices.

From the template parameter values we find mean dust extinction levels and galaxy ages given our choice of star formation histories and metallicities. The dust-free old clump is distinguished from dusty red-sequence contaminants by a high mean age of $6.2 \mathrm{Gyr}$ and a low extinction of $\left\langle E_{B-V}\right\rangle \approx 0.04$ as opposed to $3.5 \mathrm{Gyr}$ and $\left\langle E_{B-V}\right\rangle \approx 0.2$. The dusty redsequence contaminants appear with similar dust extinction levels as the blue cloud galaxies do, but with a higher galaxy age that could reflect a larger proportion of old stars. However, there could be potentially stronger extinction affecting specifically the youngest stellar population.

In Fig. 6 we show the two mean templates for the dustfree and the dusty red-sequence galaxies along with the full quantum efficiency curves of several COMBO-17 filters. In the top panel, we can see how both templates have the same $U-I$ colour, but differ in between those filters. The dusty red template is bluer at the blue end of the spectrum due to an increase of young stars. At the long-wavelength end it is redder than the dust-free old clump, presumably due to dust reddening. Comparing only the slope of the continuum between neighboring filters reveals only little difference between them. However, in the colour-colour diagrams of Fig. 5 the two types of red galaxies are more clearly differentiated because their 

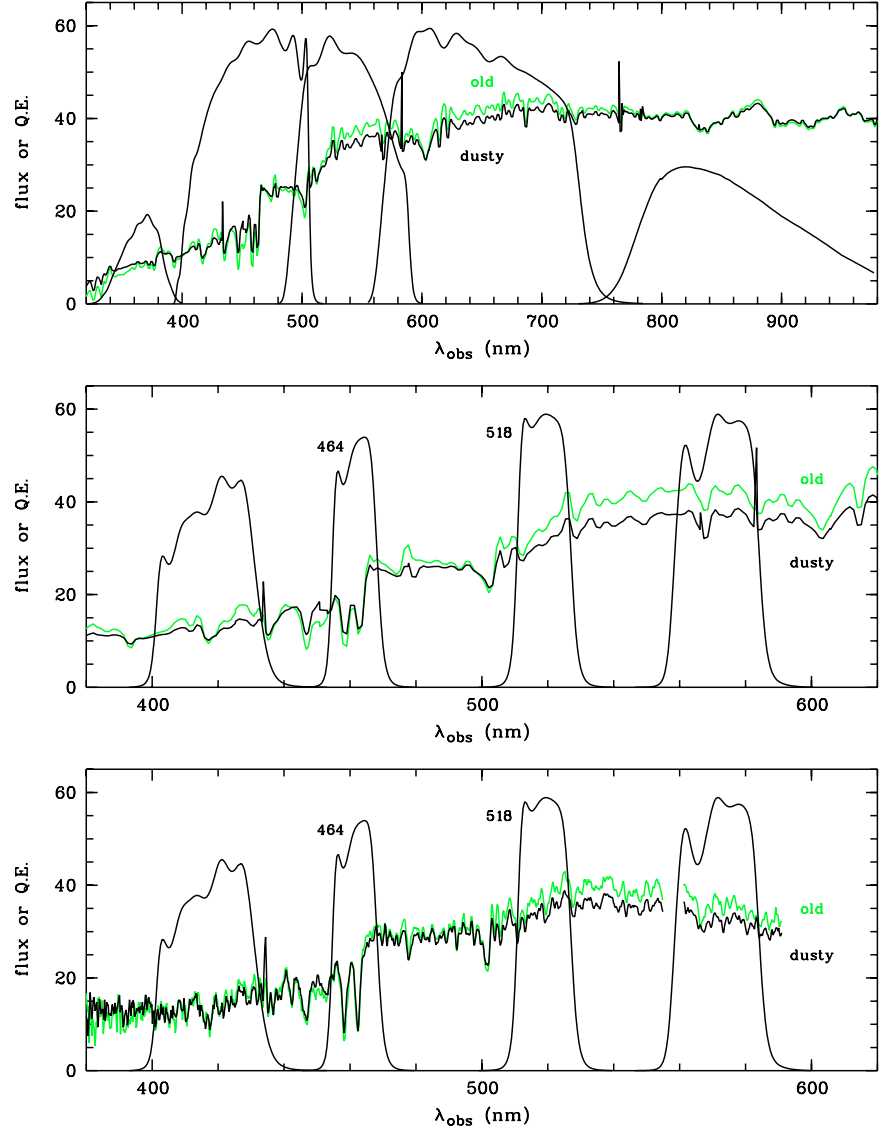

Fig. 6. Colours and spectra: top: the mean template of the dusty redsequence galaxies is redder at the red end and bluer at the blue end than that of the dust-free old clump galaxies. Middle: the dust-free old clump reflects older age in a stronger $4000 \AA$-break and $\mathrm{Ca} \mathrm{H}$ and $\mathrm{K}$-lines than the dusty red galaxies. The spectral differences in these templates match up with the mean colour differences of the two SED classes. Bottom: similar trends can be found in the mean observed spectra (we note that the flux calibration is incorrect towards the red).

principal colour difference is orthogonal to the extent of the dust-free old clump.

The middle panel shows template details around the $4000 \AA$-break and a few medium-band filters (here filter names are made from their central wavelength). Here, the templates are normalised to the flux in the 464-filter, which contains the $\mathrm{Ca} \mathrm{H}$ and $\mathrm{K}$ lines. The different template flux levels within the filters match up in fine detail to the different mean colours in the sample: when compared to the dusty red galaxies, the dustfree old clump is redder in $U-420$, bluer in 420-464, again redder in 464-518 and similar in 518-571. The detailed correspondence between template details and sample colours vindicates the use of the template grid with its age $\times$ dust structure.

The similarity in broad-band colours between old red and dusty red galaxies in such a low-redshift cluster echoes a similar ambiguity in interpreting the colours of Extremely Red Objects (EROs) found at higher redshift. These are believed to comprise dust-free, old galaxies as well as dust-reddened starforming galaxies, mostly at $z \sim 1 \ldots 2$, but also include edge-on disk galaxies at lower-redshift (Yan \& Thompson 2003).
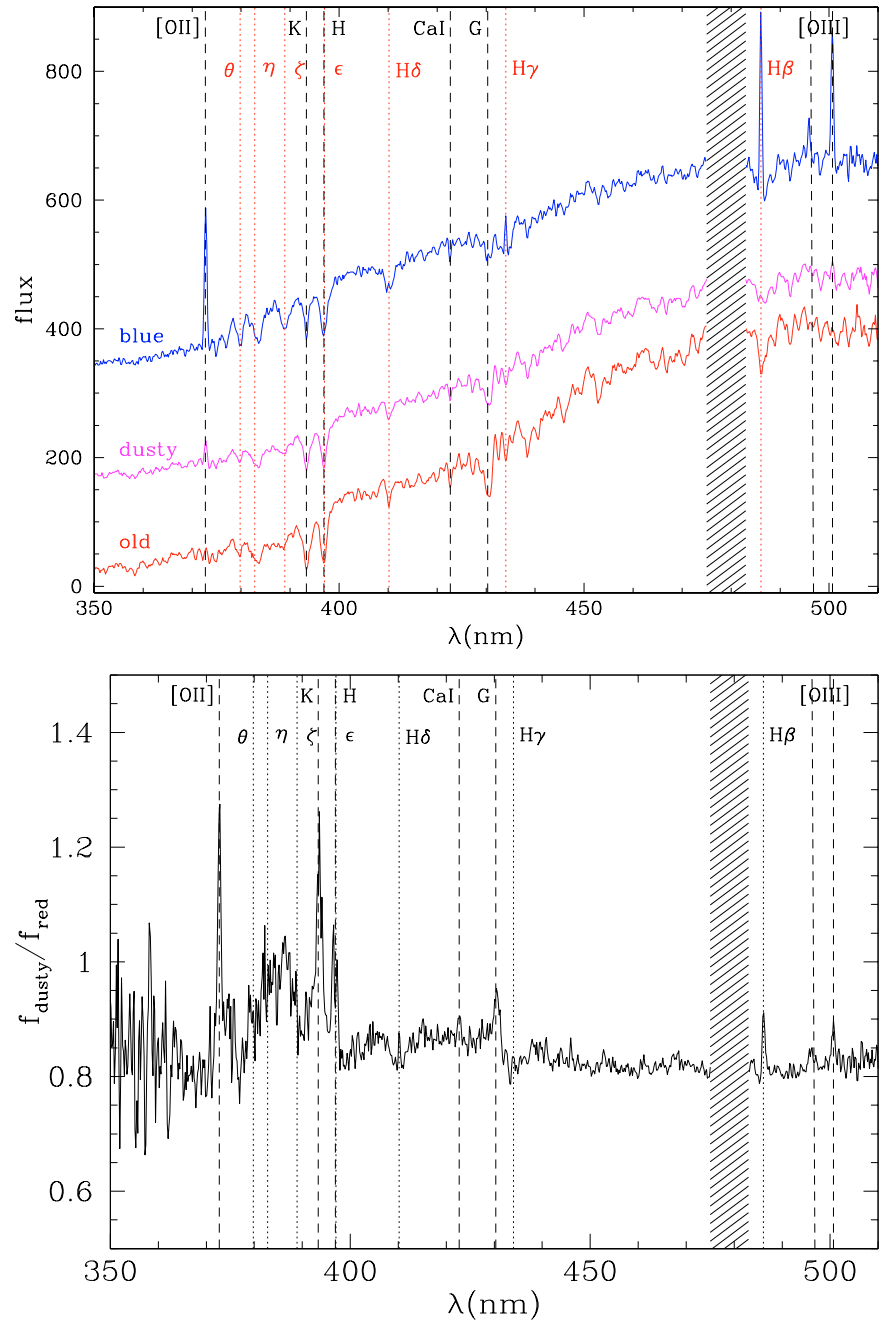

Fig. 7. Top: mean $2 \mathrm{dF}$ spectra for three galaxy types (restframe). Vertical offsets are used for clarity. The hashed area is affected by night-sky emission lines. Bottom: the mean dusty red spectrum divided by the mean old red spectrum.

\subsection{Mean spectra for three classes}

However, in order to deduce the true nature of the dusty redsequence galaxies we should investigate their observed spectra rather than the templates. We have averaged the spectra for the three types using equal weights for every galaxy. Most spectra fall into the dust-free old category because these galaxies are most abundant at high luminosity and dominate any bright flux-limited sample. With 69 objects the dusty red-sequence category contains enough data to allow a detailed comparison with galaxies from the dust-free old clump.

We show the mean spectra in Fig. 7 with relative flux offsets for clarity. From old red over dusty red to blue young galaxies, there is a clear trend of increasing Oxygen and Hydrogen emission line fluxes. Going from old red to dusty red galaxies, emission fill-in can be seen within the $\mathrm{H} \beta$ and $\mathrm{H} \delta$ absorption lines. Going again from old via dusty to blue, the $\mathrm{Ca}$ absorption lines and the G-band decrease in depth. These trends may appear quite weak when going from old red to dusty red galaxies but can be seen more clearly in the ratio spectrum of Fig. 7, 
where dusty red galaxies are divided by old red galaxies, and even weak OIII emission lines appear.

We compare the two types of red galaxies more carefully in Fig. 6. Redwards of the Ca lines, we find again the same trends first identified from the medium-band colour index 464-518 and from the templates. Bluewards of $\mathrm{Ca} \mathrm{H}$ and $\mathrm{K}$ the spectra are relatively noisy and we see less pronounced differences. The mean spectrum of dust-free old galaxies reflects an old stellar population which is passively evolving and shows no signs of ongoing star formation. We see some $\mathrm{H} \delta$-absorption which hints at recent star formation. The equivalent width of $\mathrm{H} \delta$ is $2.3 \AA$ (rest-frame), while normal cluster ellipticals are not supposed to show any clear $\mathrm{H} \delta$-lines. Measuring lines in the mean spectrum should be robust in terms of measuring the average equivalent width, but it tells us nothing about the population mix producing this average. The mean spectrum may contain a fraction of $\mathrm{k}+\mathrm{a}$ galaxies (post-starburst galaxies also known as $\mathrm{E}+\mathrm{A}$ galaxies) with pronounced $\mathrm{H} \delta$-lines.

In the dusty red-sequence galaxies we see weak OII emission as well as $\mathrm{H} \delta$-absorption, and conclude that they must be forming stars at some rate currently. Given the template solution fitting the observed colour spectrum, we find that they are moved to the red sequence via a combination of dust reddening typical of blue cloud galaxies, together with older ages typical of red-sequence galaxies. We find equivalent widths in emission of $\left\langle E W_{\mathrm{e}}(\mathrm{OII})\right\rangle=4.2 \AA$ and in absorption of $\left\langle E W_{\mathrm{a}}(\mathrm{H} \delta)\right\rangle=2.6 \AA$. In contrast, the average blue cloud galaxy has not only stronger Oxygen emission $\left(\left\langle E W_{\mathrm{e}}(\mathrm{OII})\right\rangle=17.5 \AA\right)$ but also stronger $\mathrm{H} \delta$ absorption $\left(\left\langle E W_{\mathrm{a}}(\mathrm{H} \delta)\right\rangle=4.5 \AA\right)$.

The equivalent width of $\mathrm{H} \delta$ holds clues about the recent variations in the star formation rate. Values of $E W_{\mathrm{a}}(\mathrm{H} \delta)>5 \AA$ imply that a recent starburst was followed by quenching of the star formation, such as in $\mathrm{k}+\mathrm{a}$ galaxies. If the star formation had continued, related emission would have filled in the line reducing the $E W$ (see Mercurio et al. 2004, and references therein). Dressler et al. (1999) introduced the notion of $e(a)$ galaxies, which display not only strong $\mathrm{H} \delta$ absorption as in $\mathrm{k}+\mathrm{a}$ galaxies, but also OII emission suggesting ongoing star formation. These objects are explained by Poggianti \& Wu (2000) as dust-enshrouded star bursts where age-dependent dust absorption explains the apparent contradiction from the lines: strong $\mathrm{H} \delta$ troughs arise from an A star population that has left their dusty birth place, while the very young $\mathrm{O}$ stars are still hidden such that they can not fill in the absorption with emission from their ionized environment.

At this stage, we will not offer a detailed explanation of the spectra of our class of dusty, star-forming red-sequence galaxies, nor speculate about deeply dust-enshrouded star formation. A more detailed analysis of individual spectra is the subject of another paper (Gray et al., in preparation), and Spitzer observations of this cluster field have just been obtained.

\subsection{Cluster vs. field - contamination?}

We now compare the galaxy properties between the cluster sample and the field sample in order to assess to what extent field contamination could bias the cluster results. Especially, we would like to see whether the relatively high fraction of intermediate-age dust-reddened galaxies within the redsequence of the cluster is a result of field contamination to the cluster sample.

In Fig. 8 we compare age $\times$ dust-parameters and colourmagnitude diagrams of the cluster and field samples. We find that the cluster contains an enhancement of both dust-free old populations and intermediate-age dust-reddened populations (at $\sim 3 \mathrm{Gyr}$ on our mean stellar age scale). On the other hand, the cluster shows a shortage of blue cloud galaxies, which is particularly apparent among young populations with ages of $<1$ Gyr. In the cluster, more than a third of all galaxies belong to the dust-free old clump, while in the field only $9 \%$ do. More than $20 \%$ of the cluster sample are in the redsequence despite not belonging to the dust-free old clump, while only $11 \%$ of field galaxies do. The dusty red-sequence galaxies reach higher luminosities in the cluster than in the field.

In Sect. 3.2 we reported numbers on field contamination to the cluster sample as expected from the galaxy densities measured at non-cluster redshifts. The result was a considerable contamination to the blue cloud, but in the cluster redsequence only $3 \%$ were expected to be field galaxies. This contamination rate is much below the observed contribution from dust-reddened galaxies to the cluster red-sequence. These intermediate-age dust-reddened galaxies are therefore a genuine cluster-related phenomenon, which can not be explained by effects relating to selection or contamination.

While the increased red-sequence fraction and the shortage of young vigorously star-forming galaxies in clusters has been established long ago, the increase in dust-reddened intermediate-age populations is a new trend identified here from optical data alone.

\subsection{Spatial clustering and velocity distribution}

The spatial distribution of the three galaxy types in the cluster is shown in Fig. 9. The top row shows sky maps with positions straight from the camera images. It is immediately clear that galaxies from the dust free old clump are most highly clustered, and reach the highest projected densities at the cores of the various subclusters. In contrast, dusty red galaxies and blue cloud galaxies show no high concentrations within this supercluster environment. However, we can decuce from the contamination estimate, that the total density of blue galaxies is still six times higher than average field galaxy density. The maps may suggest to the eye that both dusty red and blue galaxies trace roughly the shape of overdensities outlined by the dust-free old population, just without their strong clustering.

Going beyond 2-D projections we try to investigate the 3-D distribution in $(x, y, z)$-coordinates, although it is clear, that the $z$-coordinate reflects a combination of spatial position with peculiar velocity. The middle row in Fig. 9 shows projected maps in the $(y, z)$-plane (North is up). The strongly clustered old galaxies reveal two concentrations, a Northern group (ellipse) and a Southern group (circle), with a rest-frame velocity difference of $\Delta(c z) /(1+z) \approx 1400 \mathrm{~km} \mathrm{~s}^{-1}$ centered 

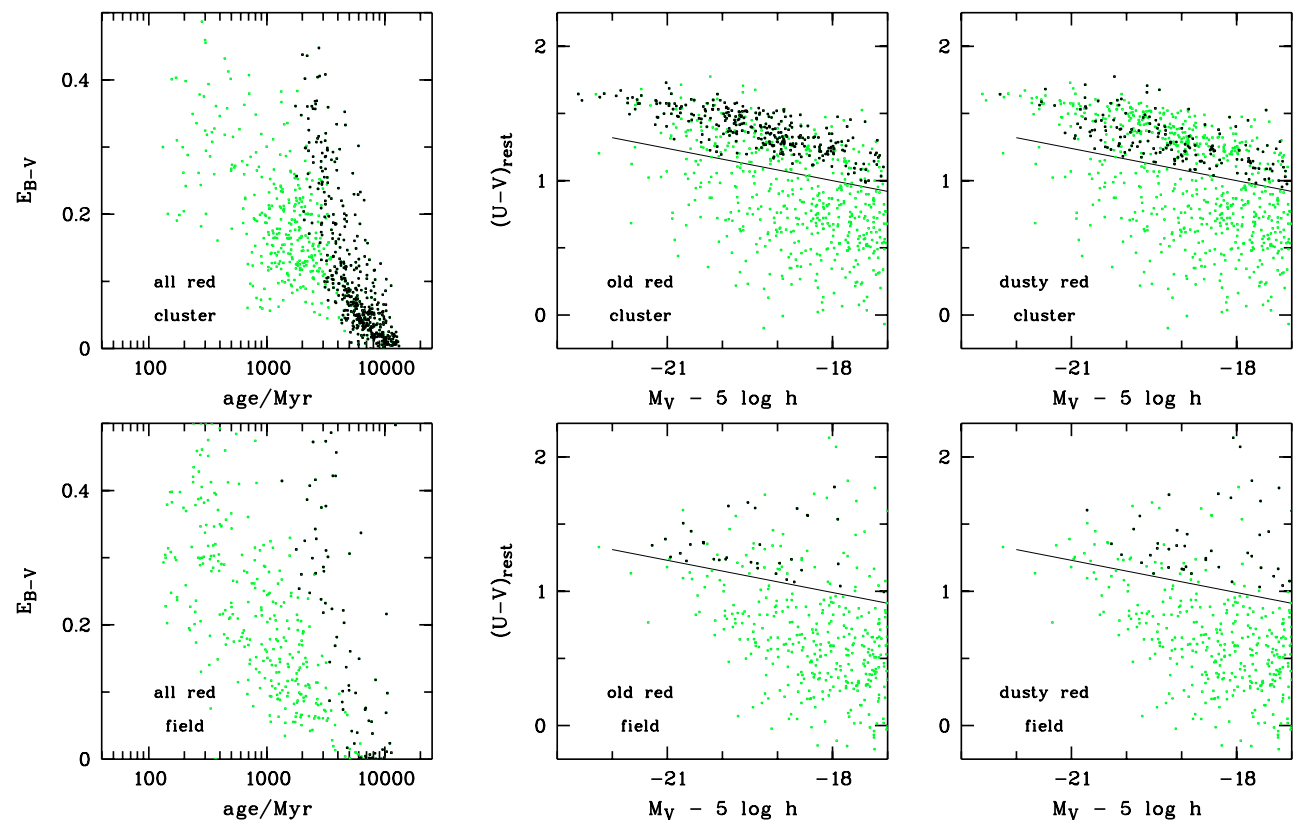

Fig. 8. Cluster (top) versus field (bottom): left: the age $\times$ dust-parameter plot shows great enhancement of red-sequence galaxies in the cluster, as well as a shortage of young $(<1 \mathrm{Gyr})$ populations. The cluster-specific enhancement applies to both, dust-free old galaxies (center) and dusty red-sequence contaminants (right). The number of objects in the cluster is roughly twice that in the field (795:385).

on $c z=49500 \mathrm{~km} \mathrm{~s}^{-1}$. There is also indication for filamentary structure. The $(y, z)$-maps can not easily show clustering as such, because galaxies in deep potential wells show wide velocity dispersions. We note, that projections in the $(x, z)$-plane happen to not reveal conspicuous structures. The distribution of dusty red galaxies follows a similar two group-structure, possibly with a slight offset to lower redshifts in the North group. The blue galaxies mostly avoid the concentrated areas which are heavily populated by red galaxies of both kinds, most notably the Northern group, and almost seem to live in the gaps left by the $(y, z)$-structure of red galaxies.

This is an intriguing piece of evidence when combined with the $(x, y)$-maps, where old red galaxies are clearly clustered, while the dusty red and blue galaxies have low clustering in common. But in the $(y, z)$-maps dusty red galaxies occupy a similar region as old red galaxies, while blue galaxies tend to avoid them. Most of the redshift signal is likely to originate from peculiar velocities, since a line-of-sight distance equal to the lateral size of our field causes only $350 \mathrm{~km} \mathrm{~s}^{-1}$ of redshift. Hence, a tentative consistent explanation of the maps could place the blue galaxies in a large low-density volume where large distances from massive cluster cores leave them with low random velocities and a natural spread in positional redshift. The old red galaxies form concentrated cluster cores that move at relative bulk velocities of $\pm 700 \mathrm{~km} \mathrm{~s}^{-1}(\mathrm{~N}+/ \mathrm{S}-)$ through the blue envelope. The dusty red galaxies would be associated in velocity and position with the cores populating their outskirts with less concentration.

Finally, we determine velocity histograms for each galaxy type independently (bottom row in Fig. 9). The full samples suggest high velocity dispersion, where especially the dusty red galaxies appear to have $\sigma_{c z} /(1+z) \approx 1200 \mathrm{~km} \mathrm{~s}^{-1}$. There are indications of a bimodal distribution among both types of red galaxies, arising from the two groups in velocity space. When analyzing only the encircled groups, we find $\sigma_{c z} /(1+z) \sim$ $550 \mathrm{~km} \mathrm{~s}^{-1}$ (rest-frame) for all four red samples. Such a split does not make sense for blue galaxies that show no structure in velocity space anyway.

However, there are differences between the North and South group: all three galaxy types in the South group have mean redshifts and velocity dispersions that are consistent with identical given our number statistics. In the North group however, we find almost no bright blue galaxies (hardly any member in the spectroscopic sample) and dusty red galaxies could be displaced to the North and to lower redshift compared to the old red galaxies. We note, that the empty regions in these $z$-diagrams are truely devoid of galaxies and not artificially empty due to selection effects. The only selection at work was a luminosity cut for the spectroscopic sample.

A complete analysis of the structure of this supercluster complex is beyond the scope of this paper. But we have learned, that the spatial and velocity distribution of the three galaxy types is consistent with dusty red galaxies forming an intermediate population whose preferred habitat are the regions where the high-density cluster cores interface with the lower-density field. The preferred habitats of old red and young blue galaxies are to either side of the interfacing dusty red galaxies.

\subsection{Type-density relation}

We now investigate how the fraction of each type among the galaxy population depends on environment, assessed by the projected galaxy density $\Sigma_{10}$. We define $\Sigma_{10}$ for any galaxy as the number of galaxies per $\square^{\prime}$ or $(\mathrm{Mpc} / h)^{2}$, within a circle whose radius is the average of the distance to the 9th and 10th nearest neighbor. In Fig. 10 these fractions are plotted 

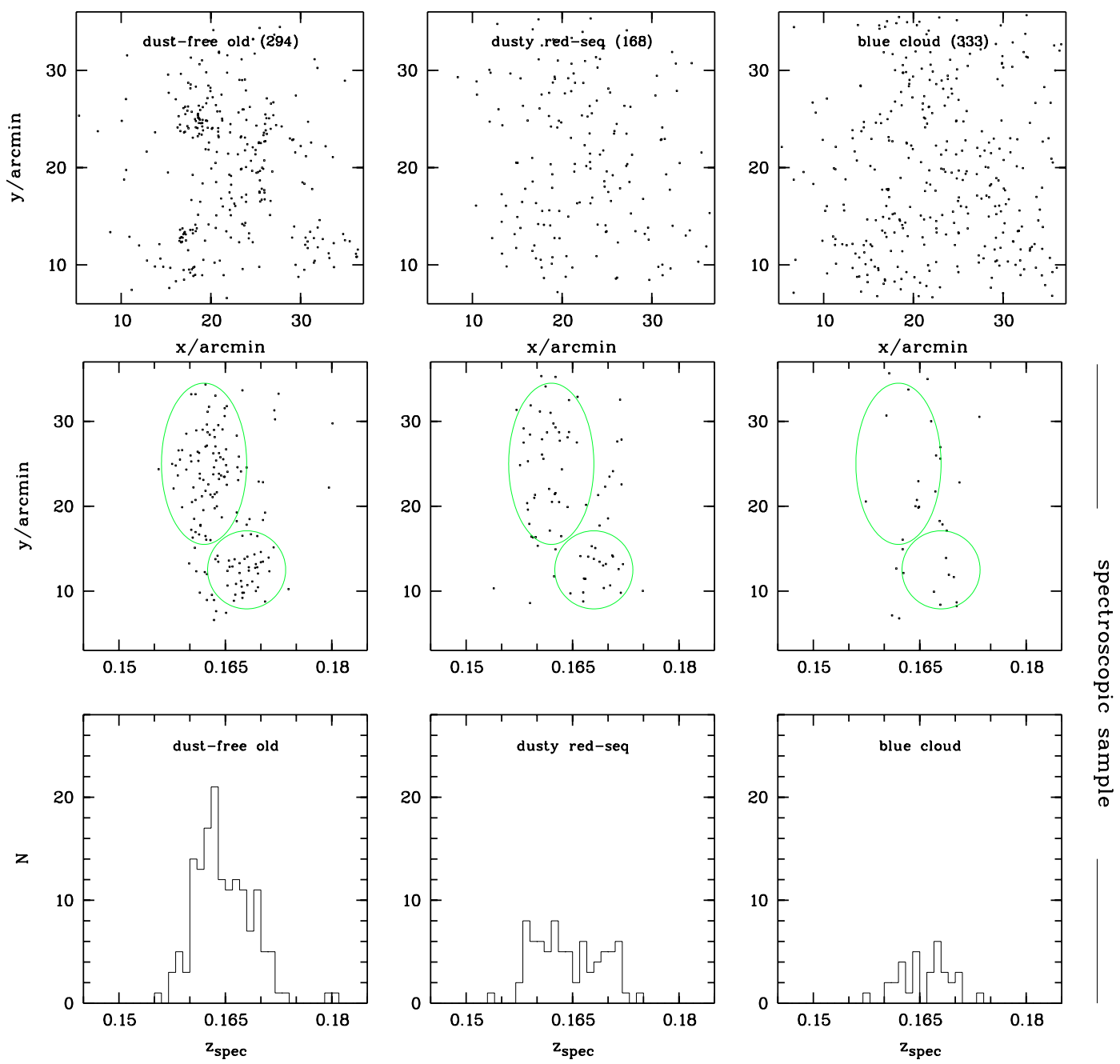

Fig. 9. Distribution of galaxy types in $(x, y, z)$ : top row: sky maps. The dust-free old galaxies are concentrated towards the cluster cores, while the other two types are more scattered over the field. Middle row: the cluster cores form two main velocity components, a North group and a South group separated by $\Delta(c z) /(1+z) \approx 1400 \mathrm{~km} \mathrm{~s}^{-1}$ in rest-frame velocity. The dusty red galaxies have a similar velocity distribution as the old red objects. Blue galaxies tend to avoid the locus of red galaxies. Bottom row: redshift distributions reflecting mostly internal dynamics.

in five bins of galaxy density, which have bin limits of $\Sigma_{10} \square^{\prime}=$ $[0.25,0.5,1,2,4,16]$. The geometric average of the whole cluster sample is $\left\langle\log \left(\Sigma_{10} \square^{\prime}\right)\right\rangle=0.222$ or $\left\langle\log \left(\Sigma_{10}(\mathrm{Mpc} / h)^{2}\right)\right\rangle=$ 2.07. In addition, we plotted the type fractions for a field sample and place the data points at $\log \left(\Sigma_{10}(\mathrm{Mpc} / h)^{2}\right)=0.67$, the mean density for the estimated level of field contamination. We remind the reader, that we have not applied any statistical subtraction of the field contamination.

Already from the cluster data alone it is clear that the fraction of dust-free old populations increases with galaxy density, while that of the blue cloud galaxies decreases. The dustreddened intermediate-age galaxies within the red-sequence appear to decline more gently towards high density than the blue cloud galaxies do. However, including the field fractions, we realize that the trend of dust-reddened red-sequence galaxies is more complex than the simple picture of old galaxies increasing and young galaxies decreasing with density. Here, we find that these dust-reddened contaminants increase strongly when going from the very low-density field environment to the medium-density region at the outskirts of the cluster, but then

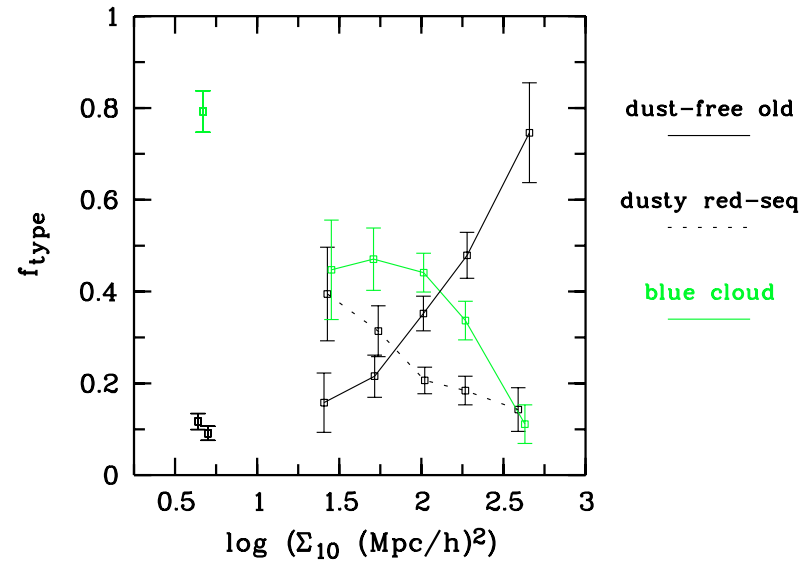

Fig. 10. Type-density relation: the cluster environment covers projected galaxy densities of $\log \left(\Sigma_{10}(\mathrm{Mpc} / h)^{2}\right)=[1.25,3.05]$. The field points lie at a density of 0.67 , but old red (9\%) and dusty red (11\%) galaxies are slightly offset for clarity. The fraction of intermediateage dust-reddened galaxies in the red-sequence increases from lowdensity fields to the outskirts of a cluster environment, but then decreases gradually with density inside the cluster. 
roll off gently towards the higher densities again. The low density gradient in their fraction renders them as an intermediate population between the blue cloud and the dust-free old clump: are these galaxies which experience a transformation from typical field to cluster properties?

\subsection{Luminosity distributions}

We determine luminosity distributions of cluster and field galaxies by type, where both have been normalised to their respective total number of galaxies at $M_{V}<-17$. The cluster is not corrected for incompleteness induced by increasing redshift errors towards the faint end, which may have shifted points by $-0.15 \mathrm{dex}$ at $M_{V} \approx-17$ if we assume $\sim 68 \%$ completeness (see Sect. 3.2). The field sample is $>90 \%$ complete at all luminosities shown here, and redshift errors do not affect its completeness given a smooth underlying field. No attempt is made to correct these observed luminosities to any sort of bolometric luminosities, e.g. by taking into account the dust extinction. Since completely obscured stellar populations would neither affect the measured luminosities nor the reddening observed in the visible stellar light, we leave any improvement involving corrections for dust until we have MIR data available.

Looking at differences between the types, we find a deficit of faint blue cloud galaxies in the cluster. The faint-end slope is not significantly different after adjusting the cluster for a roughly estimated incompleteness. This deficit is compensated by an enhancement of red-sequence galaxies at all luminosities. We find an excess of dust-free old populations at all luminosities but especially among sub-L* galaxies fainter than $M_{V}=-20$. We also find an excess of dusty red-sequence contaminants which is especially strong among galaxies brighter than $M_{V}=-19$. At the faint end of our sample dusty redsequence galaxies appear to have similar fractions in the cluster and the field sample.

The difference between the cluster and the field can be summarized with two phenomenological aspects: first, in the outskirts of the cluster a fraction of the more luminous blue cloud galaxies appear as dusty intermediate-age contaminants to the red-sequence. Here, the stellar population could be of genuinely intermediate age or it could be composed of a large old population with a smaller younger population. Secondly, in the more concentrated cluster regions a fraction of less luminous blue galaxies appear instead as red galaxies and part of the dust-free old clump, indicating that their transformation has already happened longer ago.

\section{Discussion}

\subsection{A third party: dusty red-sequence galaxies}

Recently, galaxies have been split into a red, old passively evolving and a blue, young, star-forming population. The long-established colour-density relation means red galaxies are strongly concentrated in dense cluster cores while blue galaxies overwhelmingly dominate the low-density field. In this paper we have added complexity to the simple colour-density relation from a third type of galaxies identified here to be related to clusters. They do not fit into the simple bimodal description of red-vs.-blue galaxies, and instead show signs of an ongoing transition induced by a cluster-specific mechanism. This process involves the appearance as a red-sequence galaxy which derives its colour from both dust reddening and old age of the stellar population, while actively forming stars. These properties are indicated by galaxy colours, $4000 \AA$ A-break and OII emission. Galaxies of this kind may not be restricted to the red-sequence, but in A901/902 they constitute more than a third of the red-sequence population while only $<2 / 3$ are dust-free and old.

These dusty star-forming contaminants to the red-sequence have very little in common with the dominant red-sequence population. The dusty galaxies share a spatial distribution and an avoidance of dense cluster regions with the blue cluster galaxies. But in contrast to blue galaxies, they are not common in the low-density galaxy field outside of the cluster. We may think of them as an intermediate population which could form a spatial interface between blue field galaxies and red cluster galaxies in cores.

There is further evidence that the dusty red galaxies form an intermediate population between blue and red galaxies. They contain a significant old stellar population as indicated by their $\mathrm{Ca} \mathrm{H}$ and $\mathrm{K}$ lines and $4000 \AA$-break and they are mostly of medium luminosity, at least in Abell 901/902. We repeat here, that their mean level of dust extinction is measured to be modest $\left(E_{B-V} \sim 0.2\right)$ and very similar to that in typical blue cloud galaxies.

It has been known for a long time, that a small fraction of red-sequence galaxies are edge-on spiral galaxies, which are red because we only have a clear view of their old red bulge, while their younger disk is reddened by dust lanes. So, edge-on spirals form a fraction of the dusty red galaxies in both the field and the cluster sample. However, any orientation effect can not lead to environmental differences in the fraction of edge-on spirals versus classical blue spirals. Hence, the dominant component of dusty red galaxies must be the result of an evolutionary phenomenon. Where did they come from and where do they go? Generically, we consider two possible origins:

1. either they originate from the blue cloud, their transformation was induced by some process when they got to the outskirts of the cluster, where they are now caught in the act of turning into typical old red cluster galaxies; or

2. they are minor mergers of established old cluster galaxies with infalling smaller blue field galaxies leading immediately to intermediate properties without invoking any additional process.

We will not settle any decision between these two alternatives in this paper, and we may well be looking at a mixed bag of objects from both origins plus edge-on disks. Furthermore, it is not clear that all transforming galaxies will appear unusual. If star formation just fades away as in a suffocation scenario, the galaxy may well evolve straight onto the red-sequence without any detour through a dusty, star-forming stage. We would need to know how the timescale for clearing the dust compares with the vanishing of the young stellar population to clarify this. 

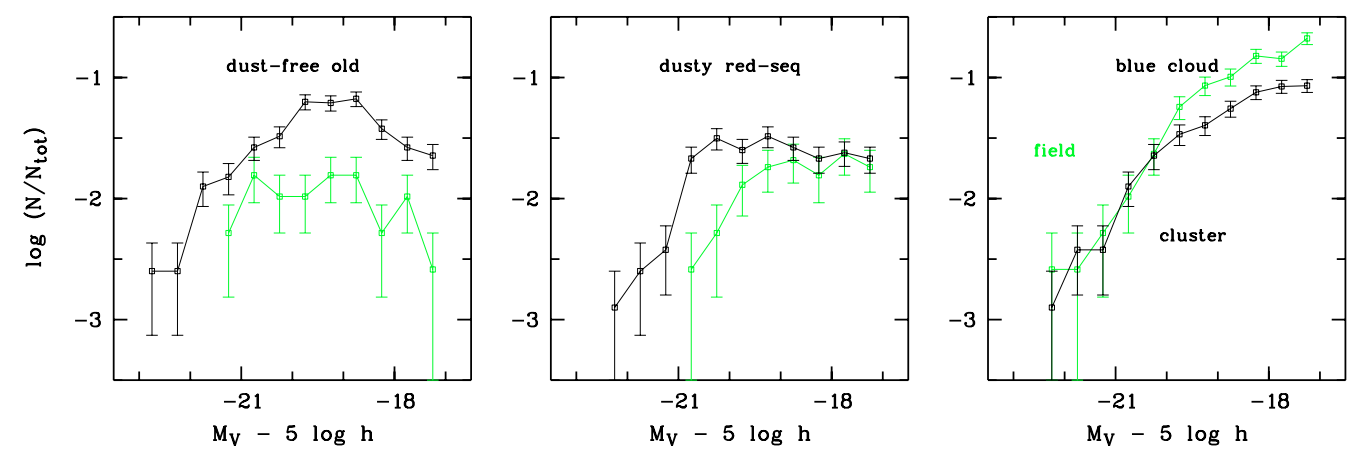

Fig. 11. Top row: luminosity distributions: the dust-free old populations follow almost a Gaussian, while the blue cloud could more easily be described by a Schechter function. The cluster shows an excess of dust-free old populations, especially fainter than $\mathrm{L}^{*}$, and of dusty redsequence contaminants, mostly brighter than $L^{*}$. The field and cluster samples are each normalised by the number of their galaxies.

However, we list some arguments supporting either one of the two scenarios:

- Pro (1): somehow galaxies in clusters have taken the turn from being blue and actively star-forming with related dust in the past to being old, red and dust-free now. Surely we should be able to catch them in the act somewhere, maybe this is it.

- Pro (1)/Contra (2): there are only few old red galaxies found in the medium-density habitat preferred by the dusty red galaxies. It seems unlikely that most red galaxies are simultaneously engaged in mergers. Figure 10 suggests that across the medium-density regime the fraction of red vs. blue galaxies remains roughly constant while the fraction of dusty red galaxies drops off towards higher density, maybe due to increased velocities deeper in the potential well making mergers harder.

- Pro (2)/Contra (1): the most luminous dusty red examples reach higher luminosities than the blue cloud field galaxies, which requires a cluster-specific high-luminosity component among the progenitors.

- Pro (2)/Contra (1): the stellar ages suggested by the template SEDs and the spectral properties show a larger proportion of old stars than expected for a typical field blue cloud galaxy which is just undergoing a first transition.

Where do they go? Most likely the dusty red galaxies will look like typical old red cluster members when their younger stars have vanished and the dust has cleared. They would not turn into typical blue galaxies again, because they already contain a significant old stellar population.

Given the abundance of galaxies of the third kind, the two possible origins translate furthermore into two routes of cluster assembly. Route (1) means, that clusters (defined by a concentration of massive old red galaxies) grow via transformation of infalling galaxies intro proper cluster galaxies, e.g. major mergers leading to dusty starbursts among infalling blue cloud galaxies. Route (2) means that clusters grow via the growth of its individual galaxies, where infalling galaxies are incorporated into existing large and red cluster galaxies via minor mergers.

We will try to collect further clues from the literature in the following. Kodama et al. (2001) have found that the dominance of blue galaxies changes into one of red galaxies at densities typical for galaxy groups rather than in cluster cores. They quote a transition density of $\Sigma_{10} \approx 10^{1.5} \mathrm{Mpc}^{-2}$ for $M_{V}<M_{V}^{*}+4, H_{0}=50 \mathrm{~km} /(\mathrm{s} \mathrm{Mpc})$ and $q_{0}=0.1$ (taking into account their Erratum on the calculation of $\Sigma$ ). In our cosmology this equates into $\Sigma_{10} \approx 10^{2.03} h^{2} \mathrm{Mpc}^{-2}$ for $M_{V} \lesssim-16.8$. We can study lower overdensities in our field thanks to the much reduced field contamination given much more accurate photo-z's. We find our dusty red galaxies at densities below the transition quoted by Kodama et al. (2001), while indeed the fraction of old red galaxies increases closer to their transition density.

While the densities where the change happens may be typical for groups, we do not see particular group signatures among the dusty red galaxies in A901. If the dusty red galaxies just formed an interface between the blue and red habitats, this would support origin (2) which requires combining progenitors from both habitats. In contrast, should a more careful analysis find our dusty red galaxies to live in groups escaping our attention here, this would support origin (1) as an internal process among the infalling blue galaxies which is only triggered by the cluster presence. Again, it remains unclear whether the dusty red galaxies in A901 mark one or the process turning blue galaxies into red galaxies. They may not tell us anything about other processes happening in groups.

\subsection{Alternative origins of the red colours}

We try to consider alternative scenarios where the colours of our third party of allegedly dusty red-sequence galaxies is not a result of dust. We consider mixed stellar populations and additional nuclear light sources:

Post-starburst galaxies of $k+a$ type: such galaxies are roughly characterized by a linear combination of an E-galaxy SED with an A-star SED. A stars are bluer than old red galaxies throughout the visual wavelength range. As a result, any sum of these components is bluer than a pure old red galaxy. Also, Balogh et al. (2005) have excluded the possibility that the colours of k+a's are significantly affected by dust. Hence, they can not show the very red colour observed at the longwavelength end in Fig. 6 which is also seen in the $R-I$ colours 
in Fig. 5. We expect $\mathrm{k}+\mathrm{a}$ galaxies to reside on the bluer end of the dust-free old clump and to some extent in the blue cloud.

Additional nuclear light sources: when considering these or indeed any internal colour gradients, we need to be aware of possible aperture bias. Our SEDs and their colours are determined from aperture photometry which probes a region of fixed physical size for any galaxy at the cluster distance. As a result, all nuclear components in the spectrum will appear enhanced as compared to SEDs from total object photometry. HST images will soon permit to quantify such effects. The origin of the nuclear component could be in dusty star-forming regions or potentially in active nuclei, and is constrained by our observations of continuum colours and emission lines. Active nuclei are almost certainly ruled out, because (i) our spectra show only very weak Oxygen lines, (ii) the objects are not detected in a $90 \mathrm{ks}$ XMM image, and (iii) the abundance of these objects. Furthermore, the continuum shape does not permit AGN light as a sole modification on top of an old red SED: the deviation from an old red SED is increasingly blue on the blue side and increasingly red on the red side.

\subsection{Previous observations of dusty star-forming cluster galaxies}

Coia et al. (2005) have discussed the properties and abundance of mid-IR sources in clusters from ISOCAM observations. Generally, MIR-luminosity is a good tracer of total star formation, given that the largest part of star formation is usually dust-enshrouded in larger galaxies. They found that half of their sources (heavily star-forming cluster galaxies) reside in the red-sequence. While they are obviously not dust-poor old populations following passive evolution, their red colour is instead explained by dust reddening. In contrast to the main red cluster population, the dusty red starbursts were not concentrated in high-density regions.

If such galaxies existed in Abell 901/902, we would identify at least the red-sequence part of the population. Most likely they are similar objects as our dusty star-forming redsequence contaminants: a property common to both is their tendency to avoid the high-density cluster cores. In the cluster $\mathrm{Cl} 0024+1654$ the MIR sources have a broader velocity dispersion than the main cluster population, just like the dusty red galaxies do in the Abell 901/902 complex. We note, that Coia et al. (2005) found red starburst galaxies associated with close neighbors in all four cases where HST imaging was available.

Poggianti \& Wu (2000) have discussed in detail a type of galaxy identified initially by Dressler et al. (1999) in distant cluster studies, which may resemble our dusty red galaxies to some extent. They have found $e(a)$ galaxies showing both OII emission and $\mathrm{H} \delta$-absorption. Using line ratios and FIR fluxes, they explained these galaxies consistently as dusty starbursts with an age-dependent extinction level. Furthermore, they found $75 \%$ of their $e(a)$ galaxies to be mergers or interacting pairs with close companions, supporting a merger origin to the starbursts.

Our dusty red galaxies show similar features, but their mean $\mathrm{H} \delta$ absorption of only $E W \sim 2.6 \AA$ does not let them pass the $e$ (a) criteria. Rather than speculating about the precise nature of our dusty star-forming red-sequence galaxies we defer this discussion until observations with HST and Spitzer have been analyzed, which will deliver a wealth of detailed information about total star formation rates and morphologies.

\section{Summary}

We have analyzed the galaxy population of the supercluster system Abell 901a, 901b and 902 located at redshift $\sim 0.17$. From the COMBO-17 catalogue, we have selected the bright galaxy population with $M_{V}<-17$ by using a photo- $z$ selection of $z=[0.155,0.185]$. Given the photo- $z$ accuracy of $<0.01$, this sample is virtually complete at $M_{V}<-19$ and could be $\sim 30 \%$ incomplete at $M_{V}=-17$. For the $\sim 250$ most luminous cluster galaxies we obtained additional spectroscopic data in the blue part of the spectrum using $2 \mathrm{dF}$. The supercluster is a system of great morphological complexity with several large concentrations which are presumably unrelaxed and will merge in the future. Our results are as follows:

1. We improve the selection of old passively evolving galaxies by using details of the COMBO-17 SED information. We find, that the red-sequence contains both galaxies which are red because they are old and passive, and those which are modestly dust-reddened (168 objects, more than $1 / 3$ of the entire red-sequence).

2. We find the long-established colour-density relation again in this system (we did not investigate the morphologydensity relation, Dressler 1980). However, the overall increase of the red galaxy fraction has two contributions, which add complexity to the colour-density relation: (a) an increase of old passively evolving galaxies in high-density cores, and (b) an excess of dusty star-forming galaxies in the medium-density outskirts.

3. Thus, we have identified a part of the galaxy population which does not follow the canonical colour-density relation. A rearticulation of the colour-density relation with three classes could be, that young blue galaxies prefer lowdensity environments, dusty red intermediate-age galaxies prefer medium-density environments and old red galaxies prefer high-density environments.

4. The fraction of dusty red-sequence galaxies among the total is much higher in the cluster $\left(\sim 22 \%\right.$ at $\left.M_{V}<-17\right)$ than in the field $(\sim 11 \%)$. Within the cluster, their fraction declines with increasing density just as the blue galaxies do. In their preferred habitat of medium-density regions they match the fraction of blue galaxies $(\sim 40 \%)$.

5. The dusty red cluster galaxies have as low spatial clustering as the blue cluster members do. However, using positionvelocity-diagrams we find that dusty red galaxies tend to be associated with the cluster cores formed by old red galaxies, while blue galaxies prefer the voids.

6. After accounting for the bimodal structure in redshift space, the velocity dispersions of dusty red and old red galaxies come out similar with $\sim 550 \mathrm{~km} \mathrm{~s}^{-1}$ for each velocity group. Looking at the supercluster as a whole, dusty red galaxies 
have the largest dispersion and preferably populate the extreme velocity ends.

7. Spectra of the dusty red-sequence galaxies show they are currently forming stars but they have plenty of old stars as well. They show mean stellar ages which are intermediate to old red and young blue galaxies. It is not clear, whether this means that (i) they are a mixture of very old stars and a much younger population, or (ii) their stars are of truely intermediate age while young stars are missing or hidden by dust.

8. The dusty red galaxies either originate from minor mergers of established old galaxies with smaller infalling blue galaxies, or result from internal transformations of infalling galaxies which is triggered by the cluster environment. They might indicate where either some or the process attacks, which transforms galaxies from blue to red.

Further observations of the Abell 901/902 field are pending. A mosaic with HST/ACS (PI Gray) will provide a wealth of morphological information and clues to the transformational mechanisms at work in the dusty red galaxies. Observations with the Spitzer will reveal hidden star formation and clarify the total star formation activity in all of the cluster galaxies. Preliminary results indicate that more than half of our red-sequence galaxies with $E_{B-V}>0.25$ and $M / M_{\odot}>10^{10}$ are detected in Spitzer $24 \mu$ images (Bell et al., in preparation).

This work has only used ground-based optical data. We used the discriminative power and concentrated information content of the medium-band survey COMBO-17 to study the cluster population with high completeness and low contamination. Just from the filter SEDs we could select cluster members and identify dusty contaminants to the red-sequence. We have thus established a third class of cluster members beyond the simple red-blue division. From previous work of the colourdensity relation it would have been counter-intuitive to predict a rich population of dusty red galaxies in higher-density environments. However, we have shown them to be a phenomenon which is genuinely related to galaxy clusters and preferentially populate their medium-density envelopes interfacing between the young blue field and the old red cores.

In the near future, many medium-band surveys which are wider or deeper than COMBO-17 will target further galaxy clusters, such as a wide-area medium-band survey with the VST. These will enlarge the sample of clusters studied with the technique presented here, and will reveal possible variations in the dusty red galaxy content, which would provide further clues to the physical origin of this phenomenon.

Acknowledgements. C.W. was supported by a PPARC Advanced Fellowship. M.E.G. was supported by a PPARC Postdoctoral Fellowship and an Anne McLaren Research Fellowship. We appreciate discussions with Alfonso Aragon-Salamanca, Michael L. Balogh,
Steven Bamford, Eric F. Bell, Mustapha Mouhcine and Stella Seitz, and thank an anonymous referee for useful comments. This publication makes use of data products from the Two Micron All Sky Survey, which is a joint project of the University of Massachusetts and the Infrared Processing and Analysis Center/California Institute of Technology, funded by the National Aeronautics and Space Administration and the National Science Foundation.

\section{References}

Baldry, I., Glazebrook, K., Brinkmann, J., et al. 2004, ApJ, 600, 681 Balogh, M. L., Bower, R. G., Smail, I., et al. 2002, MNRAS, 337, 256 Balogh, M. L., Miller, C., Nichol, R., Zabludoff, A., \& Goto, T. 2005, MNRAS, 360, 587

Barnes, J. E. 1992, ApJ, 393, 484

Bekki, K. 1999, ApJ, 510, L15

Bell, E. F., Wolf, C., Meisenheimer, K., et al. 2004, ApJ, 608, 752

Butcher, H., \& Oemler, A. 1984, ApJ, 285, 426

Coia, D., McBreen, B., Metcalfe, L., et al. 2005, A\&A, 431, 433

Colless, M., Dalton, G., Maddox, S., et al. 2001, MNRAS, 328, 1039

Connolly, A. J., Szalay, A. S., Bershady, M. A., Kinney, A. L., \& Calzetti, D. 1995, AJ, 110, 1071

Dressler, A. 1980, ApJ, 236, 351

Dressler, A., Oemler, A., Couch, W. J., et al. 1997, ApJ, 490, 577

Dressler, A., Smail, I., Poggianti, B. M., et al. 1999, ApJS, 122, 51

Fioc, M., \& Rocca-Volmerange, B. 1997, A\&A, 326, 950

Gladders, M. D., \& Yee, H. K. C. 2000, AJ, 120, 2148

Gladders, M. D., \& Yee, H. K. C. 2005, ApJS, 157, 1

Gray, M. E., Taylor, A. N., Meisenheimer, K., et al. 2002, ApJ, 568, 141

Gray, M. E., Wolf, C., Meisenheimer, K., et al. 2004, MNRAS, 347, L73

Gunn, J. E., \& Gott, J. R. 1972, ApJ, 176, 1

Kirkpatrick, J. D., Henry, T. J., \& Irwin, M. J. 1997, AJ, 113, 1421

Kodama, T., Smail, I., Nakata, F., Okamura, S., \& Bower, R. G. 2001, ApJ, 562, 9

Kroupa, P., Tout, C. A., \& Gilmore, G. 1993, MNRAS, 262, 545

Larson, R. B., Tinsley, B. M., \& Caldwell, C. N. 1980, ApJ, 237, 692

Le Fèvre, O., Vettolani, G., Paltani, S., et al. 2004, A\&A, 428, 1043

Lewis, I., Balogh, M., De Propris, R., et al. 2002, MNRAS, 334, 673

McIntosh, D. M., Zabludoff, A. I., Rix, H.-W., \& Caldwell, N. 2005, ApJ, 619, 193

Mercurio, A., Busarello, G., Merluzzi, P., et al. 2004, A\&A, 424, 79

Miller, N. A., \& Owen, F. N. 2002, AJ, 124, 2453

Moore, B., Lake, G., Quinn, T., \& Stadel, J. 1999, MNRAS, 304, 465

Pei, Y. C. 1992, ApJ, 395, 130

Poggianti, B. M., \& Wu, H. 2000, ApJ, 529, 157

Schlegel, D. J., Finkbeiner, D. P., \& Davis, M. 1998, ApJ, 500, 525

Strateva, I., Ivezic, Z., Knapp, G. R., et al. 2001, AJ, 122, 1861

Taylor, A. N., Bacon, D. J., Gray, M. E., et al. 2004, MNRAS, 353, 1176

Wolf, C., Meisenheimer, K., \& Röser, H.-J. 2001, A\&A, 365, 660

Wolf, C., Meisenheimer, K., Kleinheinrich, M., et al. 2004, A\&A, 421, 913

Yan, L., \& Thompson, D. 2003, ApJ, 586, 765 
C. Wolf et al.: Red-sequence galaxies with young stars and dust in Abell 901/902, Online Material p 1

\section{Online Material}




\section{Appendix A: Accuracy of photometric redshifts}

Spectroscopy of bright galaxies was available through the 2dFGRS survey on the S11 field with 39 galaxies including the cluster A1364 at $z \sim 0.11$ and 14 galaxies on the CDFS field, most at $R \lesssim 18$ (Colless et al. 2001). More observations on the A901 field provided a larger and deeper sample of 351 galaxies at $R \lesssim 20$ including the cluster A901/902 at $z \sim 0.165$. The total number of independent redshifts is 404 . Figure A.1 displays the redshift quality of these bright galaxies. We find that $77 \%$ of the galaxies have redshift errors $\delta_{z} /(1+z)$ below 0.01 while three objects (i.e. less than $1 \%$ ) deviate by more than $5-\sigma$ from the true redshift.

Looking specifically at the A901/2 cluster system, we notice a consistent small offset of $\left\langle\Delta_{z}\right\rangle=0.005$ between photometric and spectroscopic redshifts. The reason for such offsets lies in systematic differences between the predicted and measured photometry of the galaxies. Measurements can be off due to small photometric calibration errors, and colour predictions can be off due to slightly unrealistic template SEDs or even slightly wrong filter transmission curves. At $\lambda=600 \mathrm{~nm}$, the offset corresponds to a $2.5 \mathrm{~nm}$ error in estimating the location of spectral features from the multi-band photometry. However, another galaxy cluster, Abell 1384 lies partially within the COMBO-17 S11 field at $z \sim 0.105$ and is estimated with a larger offset of $\left\langle\Delta_{z}\right\rangle=-0.01$ in the opposite direction, suggesting that field-dependent errors in the calibration are more relevant than filter curve errors.

Spectroscopy of fainter galaxies is available via the VIMOS VLT Deep Survey (VVDS, Le Fèvre et al. 2004) on the CDFS field. Using galaxies with VVDS redshift reliability of $95 \%$ or greater, we find five outliers among 334 galaxies $(1.5 \%)$ at $R<23$ with true redshift errors on the order of 0.1 , while the remaining $98 \%$ have a distribution of photo- $z$ errors $\delta_{z}=$ $z_{\text {spec }}-z_{\text {phot }}$ with a mean offset of $\delta_{z} /(1+z)=-0.006$ and an rms scatter of $\sigma_{z} /(1+z)=0.020$.

The redshift grid for the galaxy colour library covers the range from $z=0$ to $z=1.40$ in 177 steps. These are equidistant on a $\log (1+z)$ scale with steps of 0.005 and of course limit the redshift resolution when reconstructing galaxy density features in redshift space. According to the sampling theorem, features in redshift space can be recovered if their wavelength is at least twice as large as a grid step. Thus, we have to expect that features with wavelength $\delta_{z} /(1+z)<0.01$ will be smoothed by our redshift estimation even under perfect conditions where systematic problems in photometric calibration or SED match are absent. This will not significantly restrict the power of our dataset, because our redshift estimation is probably not consistently much more accurate than 0.01 across all redshifts and SEDs we are interested in.

\section{Appendix B: In retrospect: selecting old stellar populations}

Galaxies with passively evolving old stellar populations have traditionally been selected from colour-magnitude diagrams. In the absence of any prior redshift information, observedframe colours allow to search for clusters of galaxies using a red-sequence selection (e.g., Gladders \& Yee 2000, 2005). This method capitalizes on the fact, that usually galaxies in any cluster red-sequence show redder observed-frame colours than foreground galaxies. If redshifts and rest-frame data are available, the cluster population can be separated from the field with vastly improved clarity and can include blue cluster members as well.

In rest-frame colour-magnitude diagrams (at least anywhere at $z<1$ ) the galaxy population appears bimodal with a narrow red-sequence and a wide blue cloud (Strateva et al. 2001; Baldry et al. 2004; Bell et al. 2004), provided a suitable colour index is used. The selection of an old stellar population via a red-sequence cut works best when a colour index is used that depends most sensitively on mean stellar age, i.e. a colour index made from two passbands enclosing the $4000 \AA$ A-break, such as rest-frame $U-V$. Colour indices made from two passbands which reside both on one side of this break, such as restframe $R-I$ or NUV colour indices depend to a lesser degree on age and to an increased degree on dust reddening.

We illustrate this conclusion with colour-magnitude diagrams of A901, where the location of the dust-free old clump is highlighted. Here, we would like to suppress possible errors introduced by translating measured data into rest-frame quantities and plot measured magnitudes and colours instead. At $z \sim 0.16$, the observed-frame $B-R$ corresponds approximately to rest-frame $U-V$ and $R$ to $M_{V}$ (except for a zeropoint shift). Enclosing the $4000 \AA$-break we observe the strongest possible sensitivity to age and find the red sequence at the red end of the galaxy distribution (see Fig. B.1). However, a ButcherOemler-style red-sequence cut defined by a line parallel to the colour-magnitude relation, but 0.25 bluer, still selects a number of younger, dust-reddened systems in our cluster sample.

If we use the colour index $V-I$ instead, which corresponds approximately to $(B-R)_{\text {rest }}$ the relative impact of dust reddening on the colour is increased compared to that of stellar age. As a result, the red sequence moves bluewards, further into the bulk of the galaxy population. A Butcher-Oemler style selection will now be contaminated even more by dust-reddened galaxies. However, using more detailed photometric information as in this paper helps to achieve a much cleaner selection of dust-free old galaxies. 
C. Wolf et al.: Red-sequence galaxies with young stars and dust in Abell 901/902, Online Material p 3
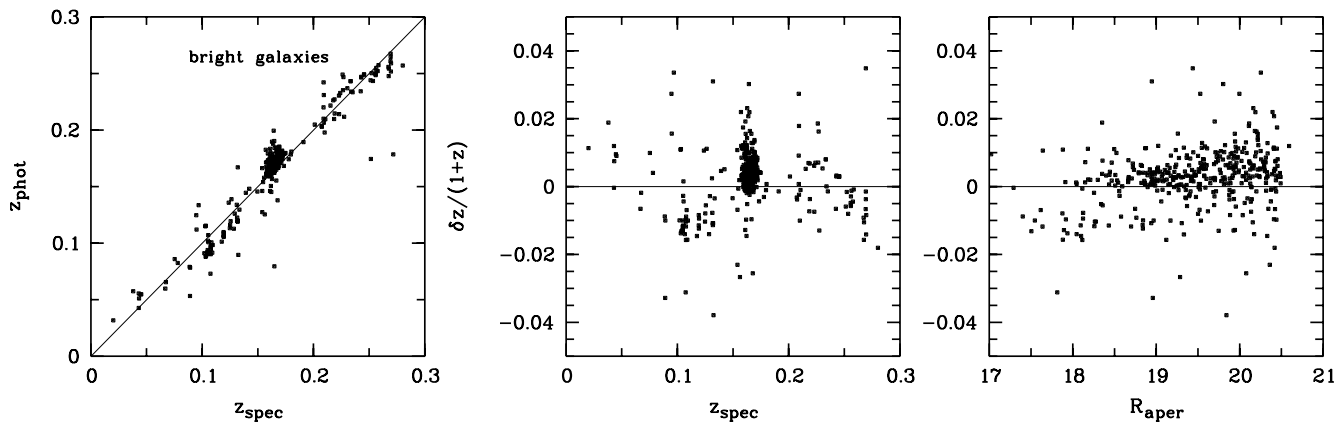

Fig. A.1. Redshift quality: 404 bright galaxies observed with 2dF on three COMBO-17 fields, mostly containing galaxies from Abell $901 / 902$. Left panel: comparison of $z_{\text {phot }}$ vs. $z_{\text {spec }}$. Center panel: redshift error $\delta z /(1+z)$ vs. redshift $z_{\text {spec }}$. Right panel: redshift error $\delta z /(1+z)$ vs. $R$-band aperture magnitude. The $1-\sigma$ redshift error is $<0.01$, and the outlier rate is $<1 \%$ ( 3 out of 404 objects with errors around 0.06 ).
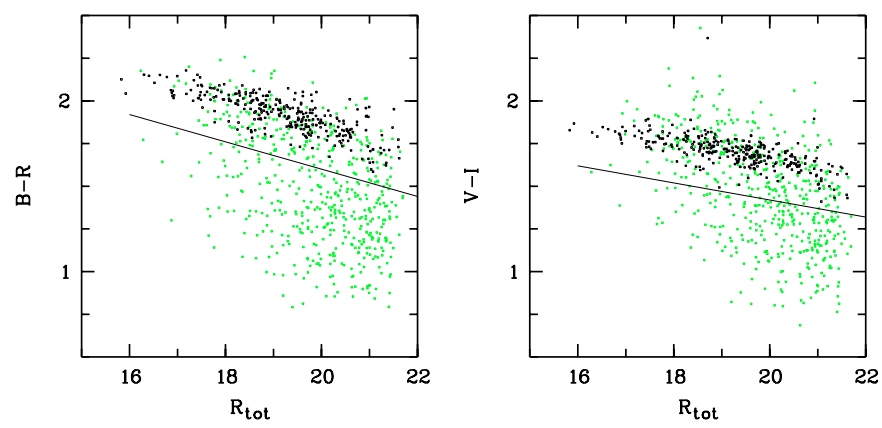

Fig. B.1. Observed-frame colour-magnitude diagrams at $z \sim 0.17$ : Left: an observed-frame colour-index straddling the 4000 Å-break makes dust-free old populations (black dots) appear as red as possible in comparison to dust-reddened galaxies. A Butcher-Oemler-style red-sequence cut (line) 0.25 below the colour-magnitude relation selects some younger dustier galaxies, which can be identified with the photometric information in COMBO-17. Right: a colour index longwards of the $4000 \AA$-break moves the red-sequence bluewards, further into the bulk of the galaxy population, making an efficient selection of dust-free old populations very challenging. 